\title{
Nonlinear Dynamics Modeling and Subharmonic Resonances Analysis of a Laminated Composite Plate
}

\author{
Ting Ma, ${ }^{1}$ Xiao Juan Song, ${ }^{2}$ and Shu Feng Lu ${ }^{1}{ }^{1}$ \\ ${ }^{1}$ Department of Mechanics, Inner Mongolia University of Technology, Hohhot 010051, China \\ ${ }^{2}$ College of Mechanical Engineering, Inner Mongolia University of Technology, Hohhot 010051, China
}

Correspondence should be addressed to Shu Feng Lu; shufenglu@163.com

Received 28 November 2019; Revised 28 January 2020; Accepted 18 May 2020; Published 13 August 2020

Academic Editor: Mohammad Rafiee

Copyright $\odot 2020$ Ting Ma et al. This is an open access article distributed under the Creative Commons Attribution License, which permits unrestricted use, distribution, and reproduction in any medium, provided the original work is properly cited.

\begin{abstract}
The nonlinear subharmonic resonance of an orthotropic rectangular laminated composite plate is studied. Based on the theory of high-order shear laminates, von Karman's geometric relation for the large deformation of plates, and Hamilton's principle, the nonlinear dynamic equations of a rectangular, orthotropic composite laminated plate subjected to the transverse harmonic excitation are established. According to the displacement boundary conditions, the modal functions that satisfy the boundary conditions of the rectangular plate are selected. The two-degree-of-freedom ordinary differential equations that describe the vibration of the rectangular plate are obtained by the Galerkin method. The multiscale method is used to obtain an approximate solution to the resonance problem. Both the amplitude-frequency equation and the average equations in the Cartesian coordinate form are obtained. The amplitude-frequency curves, bifurcation diagrams, phase diagrams, and time history diagrams of the rectangular plate under different parameters are obtained numerically. The influence of relevant parameters, such as excitation amplitude, tuning parameter, and damping coefficient, on the nonlinear dynamic response of the system is analyzed.
\end{abstract}

\section{Introduction}

Composite laminates have many advantages, such as high specific strength, high specific stiffness, and good fatigue resistance. Because laminated materials are often made into thin-walled structures, they are prone to large deformation under various external loads, resulting in nonlinear dynamic characteristics that exhibit complex geometries. Under certain excitation conditions, harmonic resonance may occur, which also has a significant impact on the accuracy of the structure. Therefore, it is necessary to analyze the nonlinear harmonic resonance characteristics of laminated composite plates.

Although the nonlinear vibration characteristics of composite structures have been studied for many years, research on the free vibration of laminated plates and shell structure still retains the attention of many scholars. Wang et al. [1] quoted a refined plate theory (RPT) with a new polynomial shape function to establish the displacement field of the middle core layer of sandwich laminates, and the free vibration frequency was obtained. Nonlinear free vibration of symmetrical magneto-electro-elastic laminated rectangular plates under the simply supported boundary condition has been studied by Razavi and Shooshtari [2]. Sadri and Younesian [3] analyzed the nonlinear free vibration of a plate-cavity system using the harmonic balance method. Aranda-Iiglesias et al. [4] studied the large amplitude axisymmetric free vibration of incompressible elastic cylinder structures. Based on the asymmetric mode shape formula of annular plates, Torabi and Ansari [5] used a pseudo-arclength continuation method to study the free vibration of carbon nanotube (CNT) reinforced composite annular plates under thermal loads.

Analytical and numerical methods have been widely used in the study of the dynamic characteristics of composite structures [6-11]. However, the solution of nonlinear forced vibration of laminated plates remains a difficult problem. For example, the reliability and convergence of the results for solving complex boundary conditions are unsatisfactory, and it is not easy to satisfy all the displacement boundary 
conditions and static boundary conditions. Khdeir and Reddy [12] solved the vibration equation of laminated plates by using the state variable method. Litewka and Lewandowski [13] studied the nonlinear vibration of Zener viscoelastic plates. Eslami and Kandil [14] studied the forced vibration of rectangular laminated composite plates subjected to harmonic excitation. Amabili [15] derived the nonlinear vibration equation of a rectangular plate by using the Lagrange equation. Delapierre et al. [16] studied the transverse nonlinear vibration of isotropic uniform annular thin films subjected to uniform transverse loads. Kumar et al. [17] carried out the nonlinear forced vibration analysis of an axially functionally graded inhomogeneous plate. Chen et al. [18] put forth the numerical solution of the nonlinear vibration of an arbitrary prestressed plate. The large amplitude forced vibration of thin rectangular plates made of different rubber materials was studied experimentally and theoretically by Balasubramanian et al. [19].

Nonlinear vibration of the laminated plates exhibits different characteristics with the change in boundary conditions. Studies on thin plates having different boundary conditions provide the following results. An analysis of the nonlinear dynamics of a clamped-clamped FGM circular cylindrical shell subjected to an external excitation and uniform temperature change was presented by Zhang et al. [20]. Bennett [21] studied the nonlinear vibration of antisymmetric angle-ply laminated plates. Rafiee et al. [22] studied the nonlinear vibration characteristics of simply supported functionally graded material shells under combined electrical, thermal, mechanical, and aerodynamic loading. Kattimani [23] studied the nonlinear vibration of composite plates and hyperbolic shells with simply supported or clamped boundary conditions. The nonlinear dynamic response of piezoelectric functionally graded material plates with different boundary conditions resting on Pasternak-type elastic foundations in the thermal environment was studied by Duc et al. [24]. Mohamed et al. [25] used a new numerical method to study the effects of axial loads, imperfections, and nonlinear elastic foundations on the natural frequencies and forced vibration characteristics of beams. Cho et al. [26] studied the vibration of rectangular plates with circular holes and stiffeners mounted on elastic devices by using the energy-based assumed mode method.

Internal resonance is also unique to nonlinear systems and is different from linear systems. Internal resonance will occur when the two natural frequencies of the system satisfy a certain relationship. The unique internal resonance phenomenon of the nonlinear system will excite the original nonexcited modes due to the energy transfer between the modes. Nayfeh and Mook [27] studied the dynamic characteristics of discrete and continuous systems under different resonance conditions. Nonlinear vibration of a composite laminated cantilever rectangular plate with oneto-one internal resonance under in-plane and transverse excitations was studied by Zhang and Zhao [28]. The nonlinear vibration behavior of carbon nanotube reinforced composite plates and piezoelectric rectangular composite laminates under parametric and forced excitations was studied by Zhang et al. [29, 30]. Chang et al. [31] studied the subharmonic responses of rectangular plates which are harmonically excited with one-to-one internal resonance. Zhang et al. [32] studied the nonlinear transverse vibrations of in-plane accelerating viscoelastic plates in the presence of principal parametric and 3:1 internal resonance.

Secondary resonance is a phenomenon particular to the nonlinear system, which includes superharmonic and subharmonic resonance. Many scholars have studied the secondary resonance of nonlinear systems. The subharmonic resonance of FGM truncated conical shell under aerodynamics and in-plane force is investigated by the method of multiple scales by Yang et al. [33]. Nonlinear subharmonic resonances of the current-conducting thin plate in electromagnetic field are studied by $\mathrm{Hu}$ and $\mathrm{Li}$ [34]. Li and Guo [35] studied the subharmonic resonance of both ends of a composite laminated circular cylindrical shell in a subsonic air flow under radial harmonic excitation by using the method of multiple scales. Jomehzadeh et al. [36] investigated the nonlinear subharmonic resonances of graphenematrix composite to the harmonic. The primary, subharmonic, and superharmonic responses of a fractional viscoelastic plate are studied by Permoon et al. [37], and a similar research has been conducted for cylindrical shells by Ahmadi and Foroutan [38]. Hosseini et al. [39] investigated the nonlinear forced vibrations of a viscoelastic piezoelectric cantilever in the cases of primary resonance and nonresonance hard excitation including subharmonic and superharmonic. Náprstek and Fischer [40] studied the super and subharmonic synchronization effects of the van der Pol equation on harmonic excitation. It is found in the literatures that most of the researches on subharmonic resonances are focused on the conservative systems having a singledegree-of-freedom system.

In this study, the subharmonic resonance characteristics of a two-degree-of-freedom laminated composite plate subjected to transverse harmonic excitations are investigated. The innovation of this paper lies in that the nonlinear vibration modeling of the thin plates with arbitrary boundary shapes and boundary conditions and the nonlinear vibration of the plates under different boundary conditions can be studied by assuming the corresponding mode function. The rectangular plate with simply supported boundary condition studied in this study is only a specific case when the boundary shape is determined and the boundary is acted on by no external force. In the absence of internal resonance, the low-order and high-order modes are uncoupled, and so they are studied separately. Based on the theory of higher-order shear deformation plate and von Karman's geometric relationship, the nonlinear dynamic equations are established by using Hamilton's principle. The ordinary differential equations for the vibration of the rectangular plate were derived by two-order discretization using the Galerkin method. The multiscale method is applied to obtain an approximate solution to the resonance problem. Both the amplitude-frequency response equation and the 
average equations in rectangular coordinates are obtained. In addition, the nonlinear dynamic responses of the twoorder modes with system parameters are compared concretely.

\section{Governing Equations of Motion}

The mechanical model of the special orthotropic symmetric rectangular laminated plate that is simply supported on four sides is shown in Figure 1. Assume the length, width, and thickness of the rectangular laminated plate to be $a, b$, and $h$, respectively, and a uniformly distributed harmonic excitation force $q=q_{0} \cos \Omega t$ is applied in the transverse plane, where $q_{0}$ is the amplitude of excitation.

The linear constitutive relation of each laminate is as follows:

$$
\begin{aligned}
& \left(\begin{array}{c}
\sigma_{x x} \\
\sigma_{y y} \\
\sigma_{x y}
\end{array}\right)^{k}=\left(\begin{array}{ccc}
Q_{11} & Q_{12} & 0 \\
Q_{12} & Q_{22} & 0 \\
0 & 0 & Q_{66}
\end{array}\right)^{k}\left(\begin{array}{l}
\varepsilon_{x x} \\
\varepsilon_{y y} \\
\gamma_{x y}
\end{array}\right)^{k}, \\
& \left(\begin{array}{c}
\sigma_{y z} \\
\sigma_{x z}
\end{array}\right)=\left(\begin{array}{cc}
Q_{44} & 0 \\
0 & Q_{55}
\end{array}\right)^{k}\left(\begin{array}{l}
\gamma_{y z} \\
\gamma_{x z}
\end{array}\right)^{k} .
\end{aligned}
$$

where $k$ represents the number of layers of the laminated plate and

$$
\begin{aligned}
& Q_{11}=\frac{E_{1}}{1-\nu_{12} \nu_{21}}, \\
& Q_{12}=\frac{\nu_{12} E_{2}}{1-\nu_{12} \nu_{21}}, \\
& Q_{22}=\frac{E_{2}}{1-v_{12} \nu_{21}}, \\
& Q_{66}=G_{12}, \\
& Q_{44}=G_{23}, \\
& Q_{55}=G_{13} .
\end{aligned}
$$

Based on the higher-order shear deformation plate theory, the displacement fields are

$$
\begin{aligned}
& u(x, y, z, t)=u_{0}(x, y, t)+z \phi_{x}(x, y, t)-z^{3} \frac{4}{3 h^{2}}\left(\phi_{x}+\frac{\partial w_{0}}{\partial x}\right), \\
& v(x, y, z, t)=v_{0}(x, y, t)+z \phi_{y}(x, y, t)-z^{3} \frac{4}{3 h^{2}}\left(\phi_{y}+\frac{\partial w_{0}}{\partial y}\right), \\
& w(x, y, z, t)=w_{0}(x, y, t),
\end{aligned}
$$

where $\left(u_{0}, v_{0}, w_{0}\right)$ represent the displacement of a point on the midplane and $\left(\phi_{x}, \phi_{y}\right)$ are the rotations of a transverse normal about the $y$ and $x$ axes, respectively.

According to the von Karman nonlinear geometric relation

$$
\begin{aligned}
& \varepsilon_{x x}=\frac{\partial u}{\partial x}+\frac{1}{2}\left(\frac{\partial w}{\partial x}\right)^{2}, \\
& \varepsilon_{x z}=\frac{1}{2}\left(\frac{\partial u}{\partial z}+\frac{\partial w}{\partial x}\right), \\
& \varepsilon_{x y}=\frac{1}{2}\left(\frac{\partial u}{\partial y}+\frac{\partial v}{\partial x}+\frac{\partial w}{\partial x} \frac{\partial w}{\partial y}\right), \\
& \varepsilon_{y y}=\frac{\partial v}{\partial y}+\frac{1}{2}\left(\frac{\partial w}{\partial y}\right)^{2}, \\
& \varepsilon_{y z}=\frac{1}{2}\left(\frac{\partial v}{\partial z}+\frac{\partial w}{\partial y}\right) .
\end{aligned}
$$

For the assumed displacement field in (3), the strains in (4) can be expressed as

$$
\begin{aligned}
\left(\begin{array}{c}
\varepsilon_{x x} \\
\varepsilon_{y y} \\
\gamma_{x y}
\end{array}\right)= & \left(\begin{array}{c}
\frac{\partial u_{0}}{\partial x}+\frac{1}{2}\left(\frac{\partial w_{0}}{\partial x}\right)^{2} \\
\frac{\partial v_{0}}{\partial y}+\frac{1}{2}\left(\frac{\partial w_{0}}{\partial y}\right)^{2} \\
\frac{\partial u_{0}}{\partial y}+\frac{\partial v_{0}}{\partial x}+\frac{\partial w_{0}}{\partial x} \frac{\partial w_{0}}{\partial y}
\end{array}\right)+z\left(\begin{array}{c}
\frac{\partial \phi_{x}}{\partial x} \\
\frac{\partial \phi_{y}}{\partial y} \\
\frac{\partial \phi_{x}}{\partial y}+\frac{\partial \phi_{y}}{\partial x}
\end{array}\right) \\
& +z^{3}\left(\begin{array}{c}
c \frac{\partial \phi_{x}}{\partial x}+c \frac{\partial^{2} w_{0}}{\partial x^{2}} \\
c \frac{\partial \phi_{y}}{\partial y}+c \frac{\partial^{2} w_{0}}{\partial y^{2}} \\
c\left(\frac{\partial \phi_{x}}{\partial y}+\frac{\partial^{2} w_{0}}{\partial x \partial y}\right)+c\left(\frac{\partial \phi_{y}}{\partial x}+\frac{\partial^{2} w_{0}}{\partial x \partial y}\right)
\end{array}\right),
\end{aligned}
$$

$$
\left(\begin{array}{l}
\gamma_{y z} \\
\gamma_{x z}
\end{array}\right)=\left(\begin{array}{c}
\phi_{y}+\frac{\partial w_{0}}{\partial y} \\
\phi_{x}+\frac{\partial w_{0}}{\partial x}
\end{array}\right)+z^{2}\left(\begin{array}{c}
3 c\left(\phi_{y}+\frac{\partial w_{0}}{\partial y}\right) \\
3 c\left(\phi_{x}+\frac{\partial w_{0}}{\partial x}\right)
\end{array}\right)
$$

where $c=-\left(4 / 3 h^{2}\right)$.

The governing equations describing the vibration of rectangular plate are obtained by the Hamilton principle:

$$
\int_{t_{1}}^{t_{2}}(\delta T-\delta U+\delta W) \mathrm{d} t=0,
$$

where the variation in potential energy $\delta U$, the variation in kinetic energy $\delta T$, and the virtual work done by the external force $\delta W$ are given by 


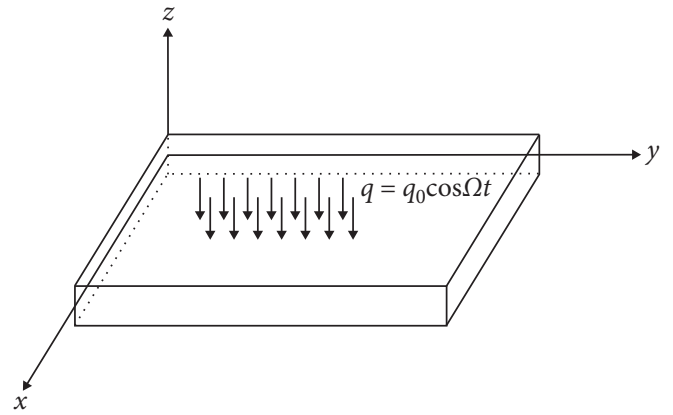

(a)

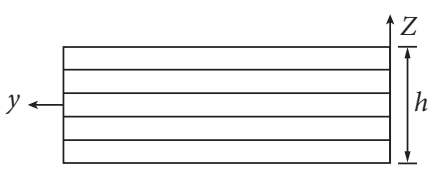

(b)

FIGURE 1: Mechanical model of a composite rectangular laminated plate.

$$
\begin{aligned}
& \delta U=\iiint\left(\sigma_{x x} \delta \varepsilon_{x x}+\sigma_{y y} \delta \varepsilon_{y y}+\sigma_{x y} \delta \gamma_{x y}+\sigma_{x z} \delta \gamma_{x z}+\sigma_{y z} \delta \gamma_{y z}\right) \mathrm{d} V \\
& \delta T= \iiint \rho \dot{w}_{0} \delta \dot{w}_{0} \mathrm{~d} V+\iiint \rho\left[\dot{u}_{0}+z \dot{\phi}_{x}+c z^{3}\left(\dot{\phi}_{x}+\frac{\partial \dot{w}_{0}}{\partial x}\right)\right] \\
& \cdot\left[\delta \dot{u}_{0}+z \delta \dot{\phi}_{x}+c z^{3}\left(\delta \dot{\phi}_{x}+\frac{\partial \delta \dot{w}_{0}}{\partial x}\right)\right] \mathrm{d} V \\
&+ \iiint \rho\left[\dot{v}_{0}+z \dot{\phi}_{y}+c z^{3}\left(\dot{\phi}_{y}+\frac{\partial \dot{w}_{0}}{\partial y}\right)\right] \\
& \cdot\left[\delta \dot{v}_{0}+z \delta \dot{\phi}_{y}+c z^{3}\left(\delta \dot{\phi}_{y}+\frac{\partial \delta \dot{w}_{0}}{\partial y}\right)\right] \mathrm{d} V
\end{aligned}
$$

$$
\begin{aligned}
\delta W= & -\iint \mu \dot{w}_{0} \delta \dot{w}_{0} \mathrm{~d} x \mathrm{~d} y+\iint q_{0} \cos \Omega t \delta w_{0} \mathrm{~d} x \mathrm{~d} y \\
& +\oint \int \widehat{\sigma}_{n n}\left[\delta u_{0 n}+z \delta \phi_{n}+c z^{3}\left(\delta \phi_{n}+\frac{\partial \delta w_{0}}{\partial n}\right)\right] \mathrm{d} z \mathrm{~d} s \\
& +\oint \int \widehat{\sigma}_{n s}\left[\delta u_{0 s}+z \delta \phi_{s}+c z^{3}\left(\delta \phi_{s}+\frac{\partial \delta w_{0}}{\partial s}\right)\right] \mathrm{d} z \mathrm{~d} s \\
& +\oint \int \widehat{\sigma}_{n z} \delta w_{0} \mathrm{~d} z \mathrm{~d} s \\
= & \oint\left(\widehat{N}_{n n} \delta u_{0 n}+\widehat{M}_{n n} \delta \phi_{n}+c \widehat{P}_{n n} \delta \phi_{n}+c \widehat{P}_{n n} \frac{\partial \delta w_{0}}{\partial n}\right. \\
& +\widehat{N}_{n s} \delta u_{0 s}+\widehat{M}_{n s} \delta \phi_{s}+c \widehat{P}_{n s} \delta \phi_{s} \\
& \left.+c \widehat{P}_{n s} \frac{\partial \delta w_{0}}{\partial s}+\widehat{Q}_{n} \delta w_{0} d s\right) \\
& +\iint q \delta w_{0} \mathrm{~d} x \mathrm{~d} y-\iint \gamma \dot{w}_{0} \delta w_{0} \mathrm{~d} x \mathrm{~d} y
\end{aligned}
$$

where $\mu$ is the damping coefficient, a superposed dot on a variable indicates its time derivative, $\rho$ is the density of the plate, $\left(\widehat{\sigma}_{n n}, \widehat{\sigma}_{n s}, \widehat{\sigma}_{n z}\right)$ are the stress components on the boundary, $\left(\delta u_{0 n}, \delta u_{0 s}\right)$ are the virtual displacements along the normal and tangential direction, respectively, on the boundary, $\left(n_{x}, n_{y}\right)$ are the direction cosines of the outward normal with respect to the $\mathrm{x}$ - and $y$-axis at a point on the plate boundary, and

$$
\begin{aligned}
& I_{i}=\int_{-h / 2}^{h / 2} \rho z^{i} \mathrm{~d} z, \\
& \left\{\begin{array}{c}
N_{x x} \\
N_{y y} \\
N_{x y}
\end{array}\right\}=\int_{-h / 2}^{h / 2}\left\{\begin{array}{c}
\sigma_{x x} \\
\sigma_{y y} \\
\sigma_{x y}
\end{array}\right\} \mathrm{d} z, \\
& \left\{\begin{array}{l}
M_{x x} \\
M_{y y} \\
M_{x y}
\end{array}\right\}=\int_{-h / 2}^{h / 2}\left\{\begin{array}{c}
\sigma_{x x} \\
\sigma_{y y} \\
\sigma_{x y}
\end{array}\right\} z \mathrm{~d} z, \\
& \left\{\begin{array}{l}
P_{x x} \\
P_{y y} \\
P_{x y}
\end{array}\right\}=\int_{-h / 2}^{h / 2}\left\{\begin{array}{c}
\sigma_{x x} \\
\sigma_{y y} \\
\sigma_{x y}
\end{array}\right\} z^{3} \mathrm{~d} z, \\
& \left\{\begin{array}{c}
Q_{y} \\
Q_{x}
\end{array}\right\}=\int_{-h / 2}^{h / 2}\left\{\begin{array}{c}
\sigma_{y z} \\
\sigma_{x z}
\end{array}\right\} \mathrm{d} z, \\
& \left\{\begin{array}{c}
R_{y} \\
R_{x}
\end{array}\right\}=\int_{-h / 2}^{h / 2}\left\{\begin{array}{c}
\sigma_{y z} \\
\sigma_{x z}
\end{array}\right\} z^{2} \mathrm{~d} z, \\
& \left\{\begin{array}{c}
\widehat{N}_{n n} \\
\widehat{N}_{n s}
\end{array}\right\}=\int_{-h / 2}^{h / 2}\left\{\begin{array}{l}
\widehat{\sigma}_{n n} \\
\widehat{\sigma}_{n s}
\end{array}\right\} \mathrm{d} z, \\
& \left\{\begin{array}{c}
\widehat{M}_{n n} \\
\widehat{M}_{n s}
\end{array}\right\}=\int_{-h / 2}^{h / 2}\left\{\begin{array}{c}
\widehat{\sigma}_{n n} \\
\widehat{\sigma}_{n s}
\end{array}\right\} z \mathrm{~d} z, \\
& \widehat{Q}_{n}=\int_{-h / 2}^{h / 2} \widehat{\sigma}_{n z} \mathrm{~d} z, \\
& \left\{\begin{array}{c}
\widehat{P}_{n n} \\
\widehat{P}_{n s}
\end{array}\right\}=\int_{-h / 2}^{h / 2}\left\{\begin{array}{c}
\widehat{\sigma}_{n n} \\
\widehat{\sigma}_{n s}
\end{array}\right\} z^{3} \mathrm{~d} z, \\
& {\left[\begin{array}{l}
\phi_{x} \\
\phi_{y}
\end{array}\right]=\left[\begin{array}{cc}
n_{x} & -n_{y} \\
n_{y} & n_{x}
\end{array}\right]\left[\begin{array}{l}
\phi_{n} \\
\phi_{s}
\end{array}\right] .}
\end{aligned}
$$

In this study, all the applied forces on the boundary are zero, That is to say, $\widehat{N}_{n n}, \widehat{N}_{n s}, \widehat{M}_{n n}, \widehat{M}_{n s}, \widehat{P}_{n n}, \widehat{P}_{n n}$ are all zeros. Substituting (7a), (7b), (7c) and (8a), (8b), (8c) into (6), the vibration equations are obtained as follows: 


$$
\begin{aligned}
& \frac{\partial N_{x x}}{\partial x}+\frac{\partial N_{x y}}{\partial y}=I_{0} \ddot{u}_{0}+\left(I_{1}+c I_{3}\right) \ddot{\phi}_{x}+c I_{3} \frac{\partial \ddot{w}_{0}}{\partial x} \\
& \frac{\partial N_{y y}}{\partial y}+\frac{\partial N_{x y}}{\partial x}=I_{0} \ddot{v}_{0}+\left(I_{1}+c I_{3}\right) \ddot{\phi}_{y}+c I_{3} \frac{\partial \ddot{w}_{0}}{\partial y} \\
& \frac{\partial\left(N_{x x}\left(\partial w_{0} / \partial x\right)\right)}{\partial x}-c \frac{\partial^{2} P_{x x}}{\partial x^{2}}+\frac{\partial\left(N_{y y}\left(\partial w_{0} / \partial y\right)\right)}{\partial y}-c \frac{\partial^{2} P_{y y}}{\partial y^{2}}+\frac{\partial\left(N_{x y}\left(\partial w_{0} / \partial y\right)\right)}{\partial x}+\frac{\partial\left(N_{x y}\left(\partial w_{0} / \partial x\right)\right)}{\partial y} \\
& -2 c \frac{\partial^{2} P_{x y}}{\partial x \partial y}+\frac{\partial Q_{x}}{\partial x}+3 c \frac{\partial R_{x}}{\partial x}+\frac{\partial Q_{y}}{\partial y}+3 c \frac{\partial R_{y}}{\partial y} \\
& =I_{0} \ddot{w}_{0}-c I_{3} \frac{\partial \ddot{u}_{0}}{\partial x}-c I_{3} \frac{\partial \ddot{v}_{0}}{\partial y}-\left(c I_{4}+c^{2} I_{6}\right) \frac{\partial \ddot{\phi}_{x}}{\partial x}-\left(c I_{4}+c^{2} I_{6}\right) \frac{\partial \ddot{\phi}_{y}}{\partial y}-c^{2} I_{6} \frac{\partial^{2} \ddot{w}_{0}}{\partial x^{2}}-c^{2} I_{6} \frac{\partial^{2} \ddot{w}_{0}}{\partial y^{2}}-q_{0} \cos \Omega t+\mu \dot{w}_{0} \text {, } \\
& \frac{\partial M_{x x}}{\partial x}+c \frac{\partial P_{x x}}{\partial x}+\frac{\partial M_{x y}}{\partial y}+c \frac{\partial P_{x y}}{\partial y}-Q_{x}-3 c R_{x} \\
& =\left(I_{1}+c I_{3}\right) \ddot{u}_{0}+\left(I_{2}+2 c I_{4}+c^{2} I_{6}\right) \ddot{\phi}_{x}+\left(c I_{4}+c^{2} I_{6}\right) \frac{\partial \ddot{w}_{0}}{\partial x}, \\
& \frac{\partial M_{y y}}{\partial y}+c \frac{\partial P_{y y}}{\partial y}+\frac{\partial M_{x y}}{\partial x}+c \frac{\partial P_{x y}}{\partial x}-Q_{y}-3 c R_{y} \\
& =\left(I_{1}+c I_{3}\right) \ddot{v}_{0}+\left(I_{2}+2 c I_{4}+c^{2} I_{6}\right) \ddot{\phi}_{y}+\left(c I_{4}+c^{2} I_{6}\right) \frac{\partial \ddot{w}_{0}}{\partial y} .
\end{aligned}
$$

Equations (9a)-(9e) can be written in form of generalized displacements $\left(u_{0}, v_{0}, w_{0}, \phi_{x}, \phi_{y}\right)$, and dimensionless parameters are introduced as $\bar{u}_{0}=\left(u_{0} / a\right), \bar{v}_{0}=\left(v_{0} / b\right)$, $\phi_{x}=\bar{\phi}_{x}, \phi_{y}=\bar{\phi}_{y}$, with the dimensionless parameter forms of other physical quantities being the same as those of [24]. Then, the dimensionless partial differential equations of vibration of the rectangular plates are obtained as

$$
\begin{aligned}
& a_{10} \frac{\partial^{2} u_{0}}{\partial x^{2}}+a_{11} \frac{\partial w_{0}}{\partial x} \frac{\partial^{2} w_{0}}{\partial x^{2}}+a_{12} \frac{\partial^{2} v_{0}}{\partial x \partial y}+a_{13} \frac{\partial^{2} w_{0}}{\partial x \partial y} \frac{\partial w_{0}}{\partial y}+a_{14} \frac{\partial^{2} u_{0}}{\partial y^{2}}+a_{15} \frac{\partial^{2} w_{0}}{\partial y^{2}} \frac{\partial w_{0}}{\partial x} \\
& =a_{16} \ddot{u}_{0}+a_{17} \ddot{\phi}_{x}+a_{18} \frac{\partial \ddot{w}_{0}}{\partial x}, \\
& b_{10} \frac{\partial^{2} u_{0}}{\partial x \partial y}+b_{11} \frac{\partial^{2} w_{0}}{\partial x \partial y} \frac{\partial w_{0}}{\partial x}+b_{12} \frac{\partial^{2} v_{0}}{\partial y^{2}}+b_{13} \frac{\partial^{2} w_{0}}{\partial y^{2}} \frac{\partial w_{0}}{\partial y}+b_{14} \frac{\partial^{2} v_{0}}{\partial x^{2}}+b_{15} \frac{\partial^{2} w_{0}}{\partial x^{2}} \frac{\partial w_{0}}{\partial y} \\
& \quad=b_{16} \ddot{v}_{0}+b_{17} \ddot{\phi}_{y}+b_{18} \frac{\partial \ddot{w}_{0}}{\partial y},
\end{aligned}
$$




$$
\begin{aligned}
c_{10} \frac{\partial^{2} u_{0}}{\partial x^{2}} \frac{\partial w_{0}}{\partial x}+c_{11}\left(\frac{\partial w_{0}}{\partial x}\right)^{2} \frac{\partial^{2} w_{0}}{\partial x^{2}}+c_{12} \frac{\partial^{2} v_{0}}{\partial x \partial y} \frac{\partial w_{0}}{\partial x}+c_{13} \frac{\partial^{2} w_{0}}{\partial x \partial} \frac{\partial w_{0}}{\partial x} \frac{\partial w_{0}}{\partial y}+c_{14} \frac{\partial^{2} w_{0}}{\partial x^{2}} \frac{\partial u_{0}}{\partial x} \\
+c_{15} \frac{\partial^{2} w_{0}}{\partial x^{2}} \frac{\partial v_{0}}{\partial y}+c_{16} \frac{\partial^{2} w_{0}}{\partial x^{2}}\left(\frac{\partial w_{0}}{\partial y}\right)^{2}+c_{17} \frac{\partial^{3} \phi_{x}}{\partial x^{3}}+c_{18} \frac{\partial^{3} \phi_{x}}{\partial x^{2} \partial y}+c_{19} \frac{\partial^{4} w_{0}}{\partial x^{4}}+c_{20} \frac{\partial^{4} w_{0}}{\partial x^{2} \partial y^{2}} \\
+c_{21} \frac{\partial^{2} u_{0}}{\partial x \partial y} \frac{\partial w_{0}}{\partial y}+c_{22} \frac{\partial^{2} v_{0}}{\partial y^{2}} \frac{\partial w_{0}}{\partial y}+c_{23}\left(\frac{\partial w_{0}}{\partial y}\right)^{2} \frac{\partial^{2} w_{0}}{\partial y^{2}}+c_{24} \frac{\partial^{2} w_{0}}{\partial y^{2}} \frac{\partial u_{0}}{\partial x}+c_{25} \frac{\partial^{2} w_{0}}{\partial y^{2}} \frac{\partial v_{0}}{\partial y} \\
+c_{26} \frac{\partial^{3} \phi_{x}}{\partial x \partial y^{2}}+c_{27} \frac{\partial^{3} \phi_{y}}{\partial y^{3}}+c_{28} \frac{\partial^{4} w_{0}}{\partial y^{4}}+c_{29} \frac{\partial^{2} v_{0}}{\partial x^{2}} \frac{\partial w_{0}}{\partial y}+c_{30} \frac{\partial^{2} w_{0}}{\partial x \partial y} \frac{\partial u_{0}}{\partial y}+c_{31} \frac{\partial^{2} w_{0}}{\partial x \partial y} \frac{\partial v_{0}}{\partial x} \\
+c_{32} \frac{\partial^{2} u_{0}}{\partial y^{2}} \frac{\partial w_{0}}{\partial x}+c_{33} \frac{\partial^{2} w_{0}}{\partial y^{2}}\left(\frac{\partial w_{0}}{\partial x}\right)^{2}+c_{34} \frac{\partial \phi_{x}}{\partial x}+c_{35} \frac{\partial^{2} w_{0}}{\partial x^{2}}+c_{36} \frac{\partial \phi_{y}}{\partial y}+c_{37} \frac{\partial^{2} w_{0}}{\partial y^{2}} \\
+c_{38} q_{0} \cos \Omega t+c_{39} \dot{w}_{0} \\
=c_{40} \ddot{w}_{0}+c_{41} \frac{\partial \ddot{u}_{0}}{\partial x}+c_{42} \frac{\partial \ddot{v}_{0}}{\partial y}+c_{43} \frac{\partial x}{\partial x}+c_{44} \frac{\partial \phi_{y}}{\partial y}+c_{45} \frac{\partial^{2} \ddot{w}_{0}}{\partial x^{2}}+c_{46} \frac{\partial^{2} \ddot{w}_{0}}{\partial y^{2}} \\
=e_{17} \ddot{v}_{0}+e_{18} \ddot{\phi}_{y}+e_{19} \frac{\partial \ddot{w}_{0}}{\partial y} . \\
e_{10} \frac{\partial^{2} \phi_{x}}{\partial x_{x}}+d_{11} \frac{\partial^{2} \phi_{y}}{\partial x \partial y}+d_{12} \frac{\partial^{3} w_{0}}{\partial x^{3}}+d_{13} \frac{\partial^{2} \phi_{x}}{\partial y^{2}}+d_{14} \phi_{x}+d_{15} \frac{\partial w_{0}}{\partial x}+d_{16} \frac{\partial^{3} w_{0}}{\partial x \partial y^{2}} \\
=d_{17} \ddot{u}_{0}+d_{18} \ddot{\phi}_{x}+d_{19} \frac{\partial \ddot{w}_{0}}{\partial x}, \\
d_{12}
\end{aligned}
$$

For the sake of convenience in writing, the transverse lines above the physical quantities are omitted. The boundary conditions of the simply supported plate can be expressed as

$$
\begin{aligned}
& x=0 \text { and } x=a, \quad v=w=N_{x x}=M_{x x}=\phi_{y}=P_{x x}=0, \\
& y=0 \text { and } y=b, \quad u=w=N_{y y}=M_{y y}=\phi_{x}=P_{y y}=0 .
\end{aligned}
$$

Due to the fact that the higher-order modes are not easily excited in structural vibration, the first two modes are taken for truncation analysis. Based on the displacement boundary conditions, the first two-order modal functions are selected as follows:

$$
\begin{aligned}
& u_{0}(x, y, t)=u_{1}(t) \cos \frac{\pi x}{a} \sin \frac{\pi y}{b}+u_{2}(t) \cos \frac{3 \pi x}{a} \sin \frac{\pi y}{b}, \\
& v_{0}(x, y, t)=v_{1}(t) \sin \frac{\pi x}{a} \cos \frac{\pi y}{b}+v_{2}(t) \sin \frac{3 \pi x}{a} \cos \frac{\pi y}{b} \\
& w_{0}(x, y, t)=w_{1}(t) \sin \frac{\pi x}{a} \sin \frac{\pi y}{b}+w_{2}(t) \sin \frac{3 \pi x}{a} \sin \frac{\pi y}{b}
\end{aligned}
$$

$$
\begin{aligned}
& \phi_{x}(x, y, t)=\phi_{1}(t) \cos \frac{\pi x}{a} \sin \frac{\pi y}{b}+\phi_{2}(t) \cos \frac{3 \pi x}{a} \sin \frac{\pi y}{b}, \\
& \phi_{y}(x, y, t)=\phi_{3}(t) \sin \frac{\pi x}{a} \cos \frac{\pi y}{b}+\phi_{4}(t) \sin \frac{3 \pi x}{a} \cos \frac{\pi y}{b} .
\end{aligned}
$$

Since the out-of-plane vibration is dominant in the vibration system, the in-plane vibrations are ignored in this study. The inertia term is ignored and the modal functions (12a)-(12e) are substituted into the vibration equations (10a)-(10e). The Galerkin method is used to separate the space-time variables, and the two-degree-offreedom ordinary differential dynamic equations are obtained as

$$
\begin{aligned}
\ddot{w}_{1}+\omega_{10}^{2} w_{1}= & \mu \dot{w}_{1}+\beta_{11} w_{2}^{3}+\beta_{22} w_{1} w_{2}^{2}+\beta_{33} w_{1}^{2} w_{2} \\
& +\beta_{44} w_{1}^{3}+P_{1} \cos (\Omega t), \\
\ddot{w}_{2}+\omega_{20}^{2} w_{2}= & \mu \dot{w}_{2}+\beta_{66} w_{1}^{3}+\beta_{77} w_{1}^{2} w_{2}+\beta_{88} w_{1} w_{2}^{2} \\
& +\beta_{99} w_{2}^{3}+P_{2} \cos (\Omega t),
\end{aligned}
$$

where the coefficients $\omega_{10}{ }^{2}, \beta_{44}, \beta_{11}, \beta_{22}, \beta_{33}, P_{1}, \omega_{20}{ }^{2}, \beta_{99}$, $\beta_{66}, \beta_{77}, \beta_{88}$, and $P_{2}$ are constants related to the system. 


\section{Perturbation Analyses}

The multiscale method is used for the approximate solution of the vibration equations. Firstly, the small parameter $\varepsilon$ is introduced, and the original equations (13a) and (13b) are transformed into

$$
\begin{aligned}
\ddot{w}_{1}+\omega_{10}{ }^{2} w_{1}= & \varepsilon \mu \dot{w}_{1}+\varepsilon \beta_{44} w_{1}^{3}+\varepsilon \beta_{11} w_{2}^{3}+\varepsilon \beta_{22} w_{1} w_{2}^{2} \\
& +\varepsilon \beta_{33} w_{1}^{2} w_{2}+P_{1} \cos (\Omega t), \\
\ddot{w}_{2}+\omega_{20}{ }^{2} w_{2}= & \varepsilon \mu \dot{w}_{2}+\varepsilon \beta_{99} w_{2}^{3}+\varepsilon \beta_{66} w_{1}^{3}+\varepsilon \beta_{77} w_{1}^{2} w_{2} \\
& +\varepsilon \beta_{88} w_{1} w_{2}{ }^{2}+P_{2} \cos (\Omega t) .
\end{aligned}
$$

The approximate solutions of (14a) and (14b) can be expressed as follows:

$$
\begin{aligned}
& w_{1}=w_{10}\left(T_{0}, T_{1}\right)+\varepsilon w_{11}\left(T_{0}, T_{1}\right), \\
& w_{2}=w_{20}\left(T_{0}, T_{1}\right)+\varepsilon w_{21}\left(T_{0}, T_{1}\right),
\end{aligned}
$$

where $T_{0}=t, T_{1}=\varepsilon t$.

The operators can be defined as

$$
\begin{aligned}
\frac{\mathrm{d}}{\mathrm{d} t}=\frac{\partial}{\partial T_{0}} \frac{\partial T_{0}}{\partial t}+\frac{\partial}{\partial T_{1}} \frac{\partial T_{1}}{\partial t}+\ldots & =D_{0}+\varepsilon D_{1}+\ldots, \\
\frac{\mathrm{d}^{2}}{\mathrm{~d} t^{2}} & =\left(D_{0}{ }^{2}+2 \varepsilon D_{0} D_{1}+\ldots,\right),
\end{aligned}
$$

where $D_{0}=\left(\partial / \partial T_{0}\right), D_{1}=\left(\partial / \partial T_{1}\right)$.

By substituting the expressions (15a), (15b) and (16a), (16b) into (14a) and (14b) and equating the coefficients of the same power of $\varepsilon$ on both sides of (14a) and (14b), one can obtain order $\varepsilon^{0}$ :

$$
\begin{aligned}
& D_{0}{ }^{2} w_{10}+\omega_{10}{ }^{2} w_{10}=P_{1} \cos (\Omega t), \\
& D_{0}{ }^{2} w_{20}+\omega_{20}{ }^{2} w_{20}=P_{2} \cos (\Omega t) .
\end{aligned}
$$

Order $\varepsilon^{1}$

$$
\begin{aligned}
& D_{0}{ }^{2} w_{11}+\omega_{10}{ }^{2} w_{11}=-2 D_{0} D_{1} w_{10}+\mu D_{0} w_{10}+\beta_{44} w_{10}{ }^{3}+\beta_{11} w_{20}{ }^{3}+\beta_{22} w_{10} w_{20}{ }^{2}+\beta_{33} w_{10}{ }^{2} w_{20}, \\
& D_{0}{ }^{2} w_{21}+\omega_{20}{ }^{2} w_{21}=-2 D_{0} D_{1} w_{20}+\mu D_{0} w_{20}+\beta_{99} w_{20}{ }^{3}+\beta_{66} w_{10}{ }^{3}+\beta_{77} w_{10}{ }^{2} w_{20}+\beta_{88} w_{10} w_{20}{ }^{2} .
\end{aligned}
$$

The solutions of (17a) and (17b) are written in the complex form:

$$
\begin{aligned}
& w_{10}=A_{1}\left(T_{1}\right) e^{i \omega_{10} T_{0}}+\bar{A}_{1}\left(T_{1}\right) e^{-i \omega_{10} T_{0}}+A_{0} e^{i \Omega T_{0}}+\bar{A}_{0} e^{-i \Omega T_{0}}, \\
& w_{20}=A_{2}\left(T_{1}\right) e^{i \omega_{20} T_{0}}+\bar{A}_{2}\left(T_{1}\right) e^{-i \omega_{20} T_{0}}+A_{3} e^{i \Omega T_{0}}+\bar{A}_{3} e^{-i \Omega T_{0}},
\end{aligned}
$$

where $A_{0}=\left(P_{1} / 2\left(\omega_{10}^{2}-\Omega^{2}\right)\right), A_{3}=\left(P_{2} / 2\left(\omega_{20}^{2}-\Omega^{2}\right)\right)$.
Subharmonic resonance occurs when the relationship between the excitation frequency and the first natural frequency of the system satisfies the relation

$$
\Omega=3 \omega_{10}+\varepsilon \sigma_{1},
$$

where $\sigma_{1}$ quantitatively describes the relationship between the frequency of the external excitation and the natural frequency of the derived system.

Substituting (19a), (19b), and (20) into (18a) and (18b) and eliminating the secular terms, the following expressions are obtained:

$$
\begin{aligned}
& \frac{\mathrm{d} A_{1}}{\mathrm{~d} T_{1}}=-\frac{i}{2 \omega_{10}}\left[\begin{array}{c}
\omega_{10} \mu A_{1} i++6 A_{1} A_{0} \bar{A}_{0} \beta_{44}+2 A_{1} A_{3} \bar{A}_{0} \beta_{33}+3 \beta_{44} A_{1}{ }^{2} \bar{A}_{1}+2 \beta_{22} A_{1} \bar{A}_{2} A_{2} \\
+2 \beta_{33} A_{1} \bar{A}_{3} A_{0}+2 \beta_{22} A_{1} \bar{A}_{3} A_{3}+\left(3 \beta_{44} \bar{A}_{1}{ }^{2} A_{0}+A_{3} \bar{A}_{1}{ }^{2} \beta_{33}\right) e^{i \sigma_{1} T_{1}}
\end{array}\right], \\
& \frac{\mathrm{d} A_{2}}{\mathrm{~d} T_{1}}=-\frac{i}{2 \omega_{20}}\left[\begin{array}{c}
\omega_{20} \mu A_{2} i++6 A_{2} A_{3} \bar{A}_{3} \beta_{99}+2 A_{2} \bar{A}_{3} A_{0} \beta_{88}+2 \beta_{88} A_{2} A_{3} \bar{A}_{0} \\
+2 \beta_{77} A_{2} \bar{A}_{0} A_{0}+3 \beta_{99} \bar{A}_{2} A_{2}{ }^{2}+2 \beta_{77} A_{2} \bar{A}_{1} A_{1}
\end{array}\right],
\end{aligned}
$$

and $A_{1}$ and $A_{2}$ are written in the polar form: 


$$
\begin{aligned}
& A_{1}\left(T_{1}\right)=\frac{1}{2} a_{1}\left(T_{1}\right) e^{i \theta_{1}\left(T_{1}\right)}, \\
& A_{2}\left(T_{1}\right)=\frac{1}{2} a_{2}\left(T_{1}\right) e^{i \theta_{2}\left(T_{1}\right)} .
\end{aligned}
$$

By substituting (22a) and (22b) into (21a) and (21b), we separate the resulting equations into the real and imaginary part, thus yielding

$$
\begin{aligned}
\dot{a}_{1}= & \frac{\mu}{2} a_{1}+\frac{\left((3 / 4) \beta_{44} a_{1}{ }^{2} A_{0}+(1 / 4) A_{3} a_{1}{ }^{2} \beta_{33}\right)}{\omega_{10}} \sin \varphi_{1}, \\
a_{1} \dot{\theta}_{1}= & -\frac{3 \beta_{44}}{\omega_{10}} A_{0}{ }^{2} a_{1}-\frac{2 \beta_{33}}{\omega_{10}} A_{0} A_{3} a_{1}-\frac{3 \beta_{44}}{8 \omega_{10}} a_{1}{ }^{3}-\frac{\beta_{22}}{4 \omega_{10}} a_{2}{ }^{2} a_{1}-\frac{\beta_{22}}{\omega_{10}} A_{3}{ }^{2} a_{1} \\
& -\frac{\left((3 / 4) \beta_{44} a_{1}{ }^{2} A_{0}+(1 / 4) A_{3} a_{1}{ }^{2} \beta_{33}\right)}{\omega_{10}} \cos \varphi_{1}, \\
\dot{a}_{2}= & \frac{\mu}{2} a_{2}, \\
\dot{\theta}_{2}= & -\frac{\beta_{77}}{\omega_{20}} A_{0}{ }^{2} a_{2}-\frac{2 \beta_{88}}{\omega_{20}} A_{0} A_{3} a_{2}-\frac{\beta_{77}}{4 \omega_{20}} a_{1}{ }^{2} a_{2}-\frac{3 \beta_{99}}{8 \omega_{20}} a_{2}{ }^{3}-\frac{3 \beta_{99}}{\omega_{20}} A_{3}{ }^{2} a_{2},
\end{aligned}
$$

where $\varphi_{1}=\sigma_{1} T_{1}-3 \theta_{1}$; equation (23c) shows that when $T_{1} \longrightarrow \infty, a_{2}$ decays to zero. That is to say, the second-order mode is not excited, and so, for the steady-state motion of the system, there is $\dot{a}_{1}=\dot{\varphi}_{1}=0$. By substituting $\dot{a}_{1}=\dot{\varphi}_{1}=0$ and $a_{2}=0$ into (23a), (23b), (23c), and (23d), the amplitudefrequency response equation of subharmonic resonance for the first-order mode is concluded:

$$
\begin{aligned}
& \left(\frac{\mu}{2} a_{1}\right)^{2}+\left(\frac{1}{3} a_{1} \sigma_{1}+\frac{3 \beta_{44}}{\omega_{10}} A_{0}{ }^{2} a_{1}+\frac{2 \beta_{33}}{\omega_{10}} A_{0} A_{3} a_{1}+\frac{3 \beta_{44}}{8 \omega_{10}} a_{1}{ }^{3}+\frac{\beta_{22}}{\omega_{10}} A_{3}{ }^{2} a_{1}\right)^{2} \\
& =\frac{\left((3 / 4) \beta_{44} a_{1}{ }^{2} A_{0}+(1 / 4) A_{3} a_{1}{ }^{2} \beta_{33}\right)^{2}}{\omega_{10}{ }^{2}} .
\end{aligned}
$$

One can also write $A_{1}$ in the form of rectangular coordinates as $A_{1}=x_{1}+x_{2} i$. Substituting $A_{1}=x_{1}+x_{2} i$ and
$A_{2}=0$ into (21a) and (21b), the average equations in the form of rectangular coordinates are obtained as

$$
\begin{aligned}
& \dot{x}_{1}=\frac{\mu}{2} x_{1}+\frac{3 A_{0}{ }^{2} \beta_{44}}{\omega_{10}} x_{2}+\frac{2 \beta_{33} A_{0} A_{3}}{\omega_{10}} x_{2}+\frac{3 \beta_{44}\left(x_{1}{ }^{2}+x_{2}{ }^{2}\right) x_{2}}{2 \omega_{10}}+\frac{A_{3}{ }^{2} \beta_{22}}{\omega_{10}} x_{2} \\
& -\frac{A_{3} \beta_{33} x_{1} x_{2}}{\omega_{10}} \cos \left(\sigma_{1} T_{1}\right)-\frac{3 A_{0} \beta_{44} x_{2}{ }^{2}}{2 \omega_{10}} \sin \left(\sigma_{1} T_{1}\right)+\frac{A_{3} \beta_{33} x_{1}{ }^{2}}{2 \omega_{10}} \sin \left(\sigma_{1} T_{1}\right) \\
& -\frac{A_{3} \beta_{33} x_{2}{ }^{2}}{2 \omega_{10}} \sin \left(\sigma_{1} T_{1}\right)-\frac{3 A_{0} \beta_{44} x_{1} x_{2}}{\omega_{10}} \cos \left(\sigma_{1} T_{1}\right)+\frac{3 A_{0} \beta_{44} x_{1}{ }^{2}}{2 \omega_{10}} \sin \left(\sigma_{1} T_{1}\right) \text {, } \\
& \dot{x}_{2}=\frac{\mu}{2} x_{2}-\frac{3 A_{0}{ }^{2} \beta_{44}}{\omega_{10}} x_{1}-\frac{2 \beta_{33} A_{0} A_{3}}{\omega_{10}} x_{1}-\frac{3 \beta_{44}\left(x_{1}{ }^{2}+x_{2}{ }^{2}\right) x_{1}}{2 \omega_{10}}-\frac{A_{3}{ }^{2} \beta_{22}}{\omega_{10}} x_{1} \\
& -\frac{3 \beta_{44} A_{0} x_{1} x_{2}}{\omega_{10}} \sin \left(\sigma_{1} T_{1}\right)-\frac{\beta_{33} A_{3} x_{1} x_{2}}{\omega_{10}} \sin \left(\sigma_{1} T_{1}\right)-\frac{3 \beta_{44} A_{0} x_{1}^{2}}{2 \omega_{10}} \cos \left(\sigma_{1} T_{1}\right) \\
& +\frac{3 \beta_{44} A_{0} x_{2}{ }^{2}}{2 \omega_{10}} \cos \left(\sigma_{1} T_{1}\right)-\frac{\beta_{33} A_{3} x_{1}{ }^{2}}{2 \omega_{10}} \cos \left(\sigma_{1} T_{1}\right)+\frac{\beta_{33} A_{3} x_{2}{ }^{2}}{2 \omega_{10}} \cos \left(\sigma_{1} T_{1}\right) \text {. }
\end{aligned}
$$


The external excitation frequency and second natural frequency of the system satisfy the following relationship:

$$
\Omega=3 \omega_{20}+\varepsilon \sigma_{1} .
$$

Similar to the first order, the amplitude-frequency response equation of subharmonic resonance for the secondmode mode is given by

$$
\begin{gathered}
\left(\frac{\mu}{2} a_{2}\right)^{2}+\left(\frac{1}{3} a_{2} \sigma_{1}+\frac{\beta_{77}}{\omega_{20}} A_{0}{ }^{2} a_{2}+\frac{2 \beta_{88}}{\omega_{20}} A_{0} A_{3} a_{2}+\frac{3 \beta_{99}}{8 \omega_{20}} a_{2}{ }^{3}+\frac{3 \beta_{99}}{\omega_{20}} A_{3}{ }^{2} a_{2}\right)^{2} \\
=\frac{\left((1 / 4) \beta_{88} A_{0} a_{2}{ }^{2}+(3 / 4) \beta_{99} A_{3} a_{2}{ }^{2}\right)^{2}}{\omega_{20}{ }^{2}} .
\end{gathered}
$$

The average equations in the form of rectangular coordinates of the subharmonic resonance for the second mode can be obtained as follows:

$$
\begin{aligned}
\dot{x}_{3}= & -\frac{\beta_{88} A_{0} x_{3} x_{4}}{\omega_{20}} \cos \left(\sigma_{1} T_{1}\right)+\frac{A_{0}{ }^{2} \beta_{77}}{\omega_{20}} x_{4}+\frac{3 A_{3}{ }^{2} \beta_{99}}{\omega_{20}} x_{4}+\frac{2 \beta_{88} A_{0} A_{3}}{\omega_{20}} x_{4} \\
& +\frac{3 \beta_{99}\left(x_{3}{ }^{2}+x_{4}{ }^{2}\right) x_{4}}{2 \omega_{20}}+\frac{\mu}{2} x_{3}-\frac{3 A_{3} \beta_{99} x_{4}^{2}}{2 \omega_{20}} \sin \left(\sigma_{1} T_{1}\right)+\frac{3 A_{3} \beta_{99} x_{3}^{2}}{2 \omega_{20}} \sin \left(\sigma_{1} T_{1}\right) \\
& -\frac{A_{0} \beta_{88} x_{4}{ }^{2}}{2 \omega_{20}} \sin \left(\sigma_{1} T_{1}\right)+\frac{A_{0} \beta_{88} x_{3}{ }^{2}}{2 \omega_{20}} \sin \left(\sigma_{1} T_{1}\right)-\frac{3 \beta_{99} A_{3} x_{3} x_{4}}{\omega_{20}} \cos \left(\sigma_{1} T_{1}\right), \\
\dot{x}_{4}= & -\frac{3 A_{3} \beta_{99} x_{3} x_{4}}{\omega_{20}} \sin \left(\sigma_{1} T_{1}\right)-\frac{3 \beta_{99}\left(x_{3}{ }^{2}+x_{4}{ }^{2}\right) x_{3}}{2 \omega_{20}}+\frac{\beta_{88} A_{0} x_{4}{ }^{2}}{2 \omega_{20}} \cos \left(\sigma_{1} T_{1}\right) \\
& -\frac{A_{0}{ }^{2} \beta_{77} x_{3}-\frac{2 \beta_{88} A_{0} A_{3}}{\omega_{20}} x_{3}-\frac{3 A_{3}{ }^{2} \beta_{99}}{\omega_{20}} x_{3}+\frac{\mu}{2} x_{4}+\frac{3 A_{3} \beta_{99} x_{4}{ }^{2}}{2 \omega_{20}} \cos \left(\sigma_{1} T_{1}\right)}{2 \omega_{20}} \cos \left(\sigma_{1} T_{1}\right)-\frac{\beta_{88} A_{0} x_{3}{ }^{2}}{2 \omega_{20}} \cos \left(\sigma_{1} T_{1}\right) . \\
& -\frac{A_{0} \beta_{88} x_{3} x_{4}}{\omega_{20}} \sin \left(\sigma_{1} T_{1}\right)-\frac{3 A_{3} \beta_{99} x_{3}^{2}}{2 \omega_{20}}
\end{aligned}
$$

\section{Results and Discussion}

The values of parameters related to the laminated materials are, respectively, $\beta_{11}=53.07, \quad \beta_{22}=-2.93, \quad \beta_{33}=98.4$, $\beta_{44}=-80.36, \quad \beta_{66}=-6.36, \quad \beta_{77}=12.07, \quad \beta_{88}=200.5$, $\beta_{99}=-180.57, \omega_{10}=5.12, \omega_{20}=10.6$. Based on the amplitude-frequency equations (24) and (27) of the two modes, the effects of damping, excitation amplitude, and excitation frequency on the $1 / 3$ subharmonic resonance characteristics are studied, as shown in Figures 2-4.

Figure 2 is the amplitude-frequency response curve of the two-order modes of the rectangular laminated plate for different excitation amplitudes when $1 / 3$ subharmonic resonance occurs. The vertical coordinate represents the amplitude of two-order modes, and the abscissa is the detuning parameter. $P_{1}$ and $P_{2}$ are the amplitudes of external excitation. It can be seen from Figure 2 that there are two steady-state solutions for the two-order modes, respectively, at the same excitation frequency. When the excitation amplitudes are the same, the amplitudes of the two steady-state solutions increase gradually with the increase in excitation frequency. When the excitation frequency is the same, the influence of the excitation amplitude on the steady-state solutions with small vibration amplitude of the two-order modes is significant. The solution with small amplitude of the first-order mode increases with the increase in the excitation amplitude. Contrary to the first-order mode, the solution with small amplitude of the second-order mode decreases with the increase in the excitation amplitude. For the same excitation frequency, the influence of the excitation amplitude on steady-state solutions with the large amplitude of the two-order modes is not obvious.

Figure 3 shows the amplitude-frequency response curves of the two-order modes of the rectangular laminated plate for different damping values when $1 / 3$ subharmonic resonance occurs. The vertical coordinate is the vibration amplitude of the two-order modes, and the abscissa is the detuning parameter. $\mu$ is a physical quantity that characterizes the damping coefficient of laminated materials. Obviously, it can be seen from Figure 3 that the damping 

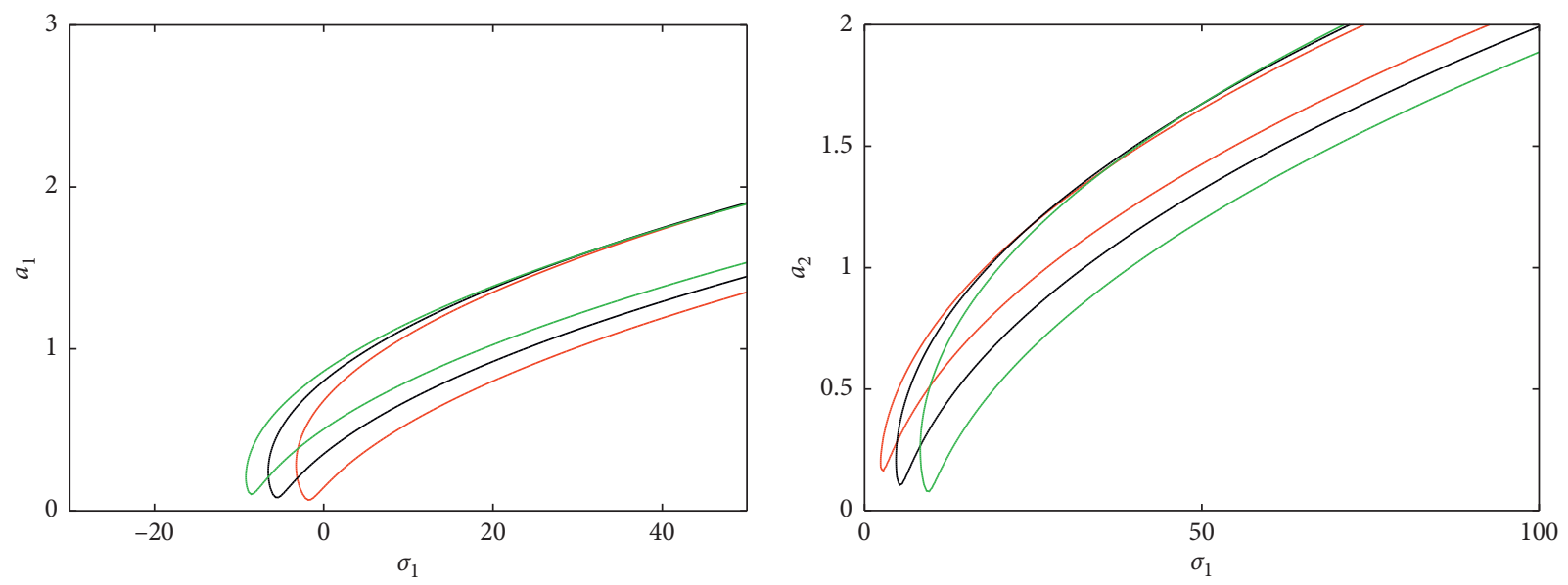

$$
\begin{aligned}
P_{1} & =10 \\
-P_{2} & =25 \\
-P_{3} & =40
\end{aligned}
$$

$$
\begin{array}{r}
P_{2}=200 \\
-P_{2}=300
\end{array}
$$

(a)

(b)

FIgURe 2: Amplitude-frequency response curves of the first-order and second-order mode for different excitation amplitudes.

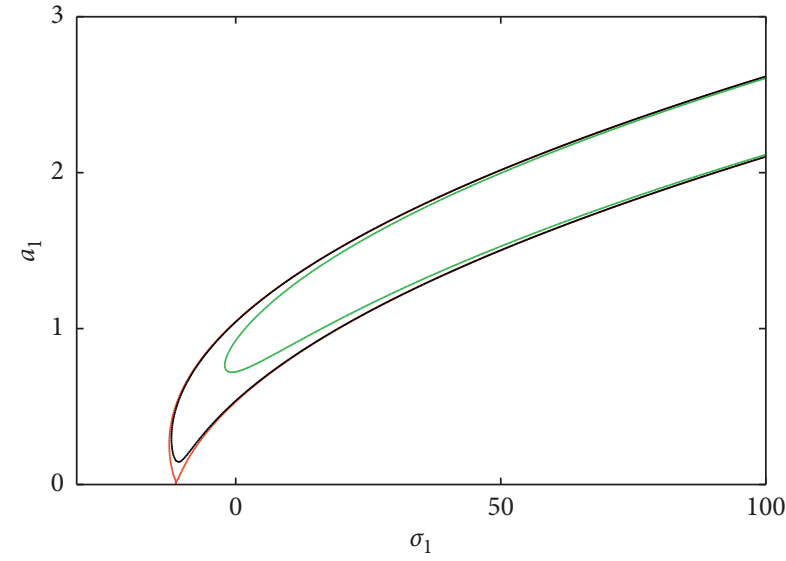

$$
\begin{aligned}
-\mu & =0.05 \\
-\mu & =1 \\
\mu & =5
\end{aligned}
$$

(a)

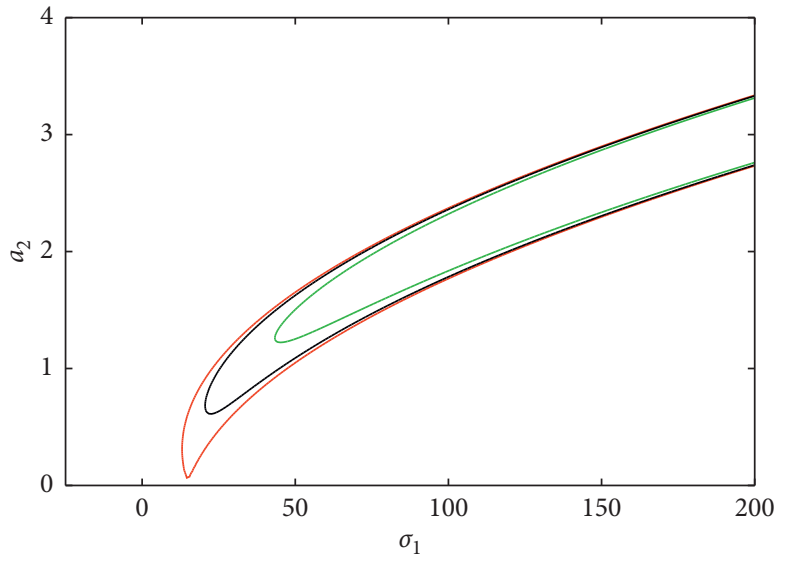

$\begin{aligned} \mu & =0.5 \\ \mu & =5 \\ \mu & =10\end{aligned}$

Figure 3: Amplitude-frequency response curves of the first-order and the second-order mode for different damping coefficients.

affects the range of the steady-state solution (i.e., distribution region of the curve). As the damping value increases, the range within which the solution of the subharmonic vibration exists becomes smaller, while the two modal amplitudes are also affected by the damping coefficient. With the change in damping, the overall change trend of the amplitude-frequency response curve of the two-order modes of the laminated plates is consistent.

Figure 4 shows ptthe force-amplitude response curves of the two-order modes of a rectangular laminated plate under different external excitation frequencies when $1 / 3$ subharmonic resonance occurs. The vertical coordinate represents the vibration amplitude of two-order modes, and the abscissa is the external excitation amplitude. It can be seen from Figure 4 that there are also two nonzero steady-state solutions for the two modes, respectively. Under the same external excitation frequency, the steady-state solutions associated with small amplitudes first decrease and then increase with the increase in the excitation amplitude, and the solutions with large amplitude decrease monotonously as the excitation amplitude increases. For the same external excitation amplitude, as the excitation frequency increases, the vibration amplitudes of the two modes also increase. The trend of the force-amplitude response curves of the two-order modes of the laminated plate remains identical. Besides, from the analysis of the frequency-resonance curves, it can be found that the results of this paper are consistent with the qualitative results given by $\mathrm{Hu}$ and $\mathrm{Li}$ [28] and Permoon et al. [37]. 


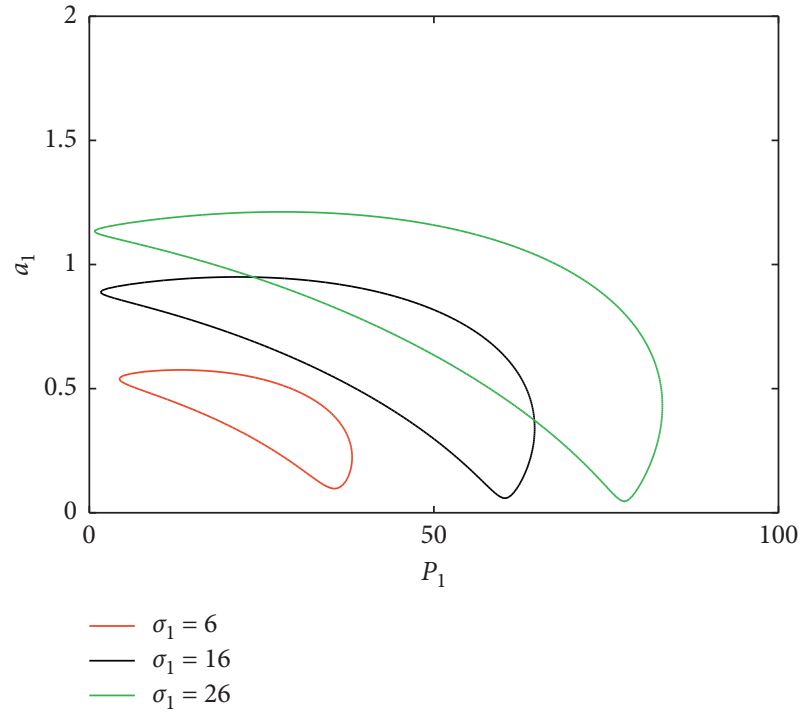

(a)

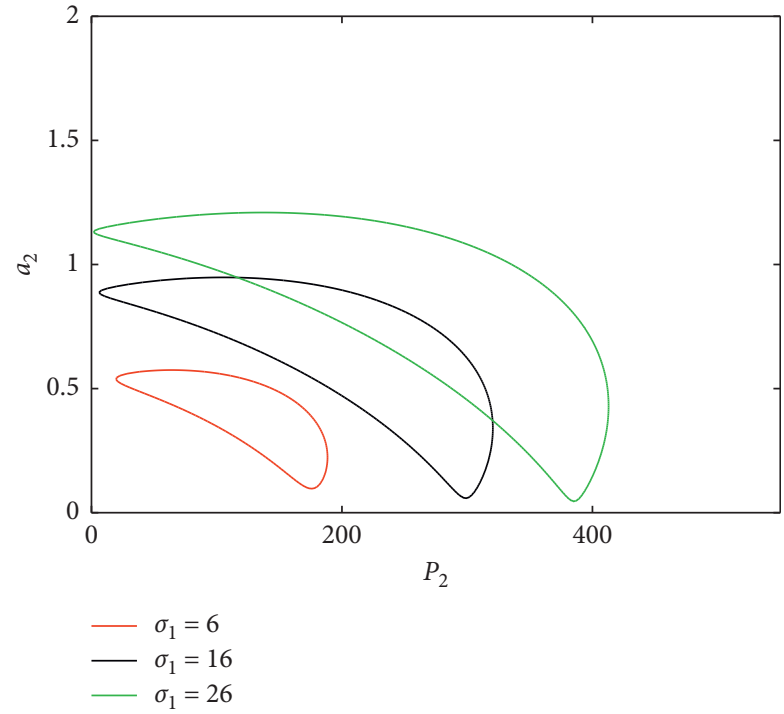

(b)

FIGURE 4: Force-amplitude response curves of the first-order and second-order mode for different excitation frequencies.

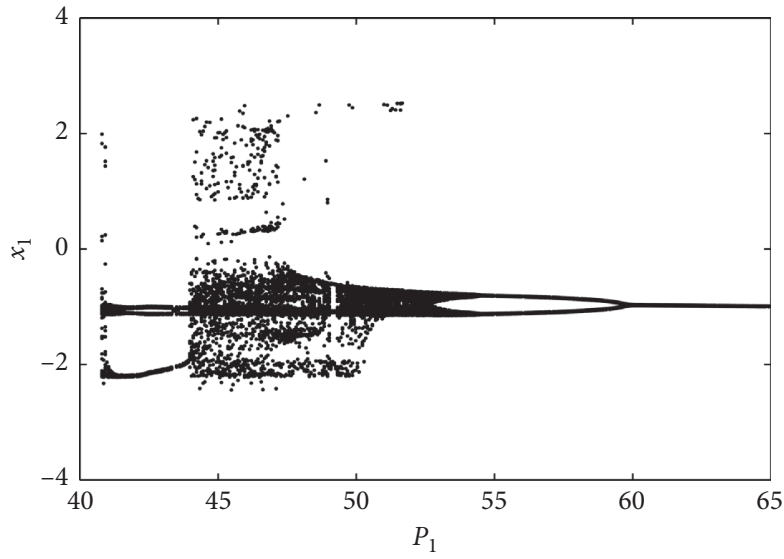

Figure 5: Bifurcation diagram for the first-order mode via external excitation.

Based on the average (25a) and (25b), the bifurcation diagram for the first-order vibration is simulated numerically by the Runge-Kutta method. The amplitude of the external excitation is selected as the control parameter of the system, and the influence of the amplitude of the external excitation on the nonlinear vibration characteristics of the system is studied. The initial conditions are $x_{10}=0.8$, $x_{20}=1.1$, and the other parameters related to the laminate materials are the same as those in the above amplitudefrequency equation (24). The global bifurcation diagram of the subharmonic vibration of the rectangular laminated plate is obtained as shown in Figure 5.

In Figure 5, the abscissa is the amplitude of the external excitation, and the vertical coordinate is the abstract physical quantity that represents the transverse deflection of the rectangular plate. It can be seen from Figure 5 that for a small amplitude of the external excitation, the vibration response of the first mode of the subharmonic resonance of the laminated plate is period-2 motion. As the amplitude of external excitation increases, the vibration response of the system changes from periodic motion to chaotic motion. With the continual increase in the amplitude of the external excitation, the response of the system changes from chaotic motion to quasiperiodic motion and subsequently to period-2 motion. Finally, the vibration response of the system changes to haploid periodic motion. In the brief interval when the amplitude of external excitation changes, the first-order mode undergoes all the forms of vibration of the nonlinear response. It shows a variety of dynamic characteristics. At the same time, the phase and waveform diagrams of different vibration states are also obtained. Figures 6-10 show the waveforms and phase diagrams of the subharmonic vibration responses of rectangular laminated plates under different vibration stages in Figure 5. 


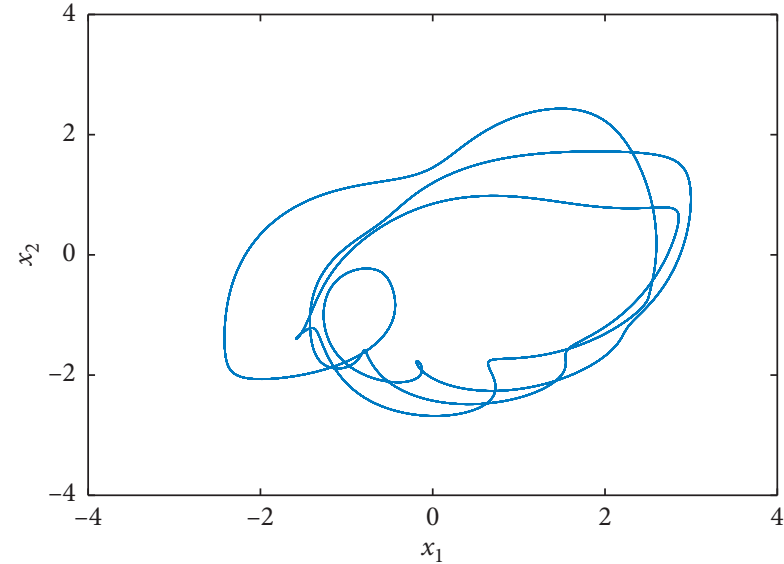

(a)

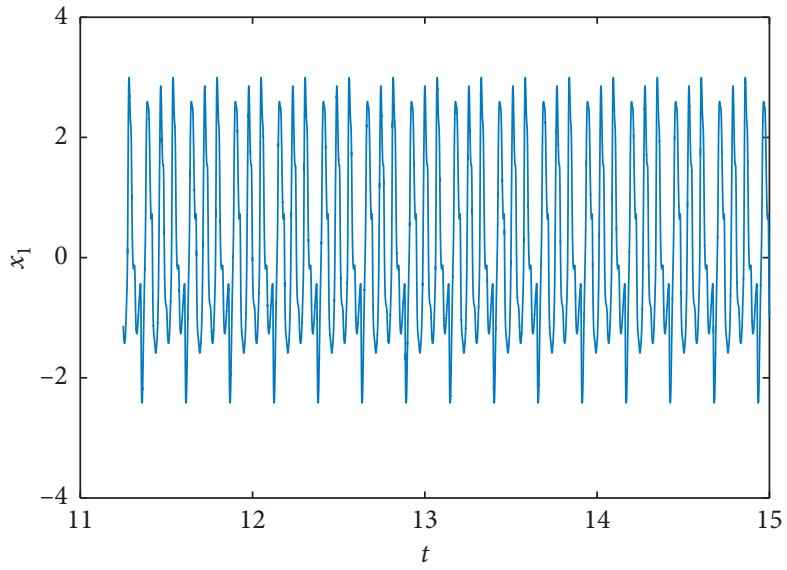

(b)

Figure 6: Phase portrait on plane $\left(x_{1}, x_{2}\right)$ and the waveforms on the plane $\left(t, x_{1}\right)$ of the first-order mode of the system with periodic-3 motion when $P_{1}=42$.

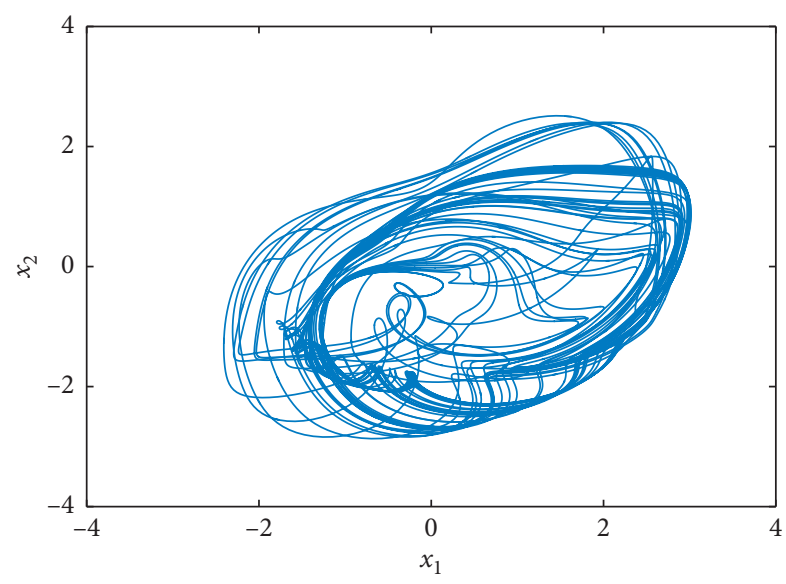

(a)

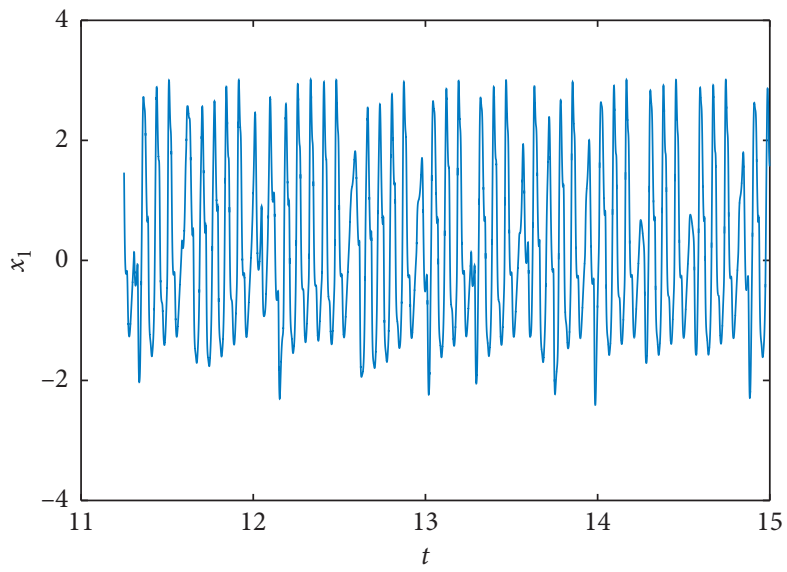

(b)

Figure 7: Phase portrait on plane $\left(x_{1}, x_{2}\right)$ and the waveforms on the plane $\left(t, x_{1}\right)$ of the first-order mode of the system with chaotic motion when $P_{1}=46$.

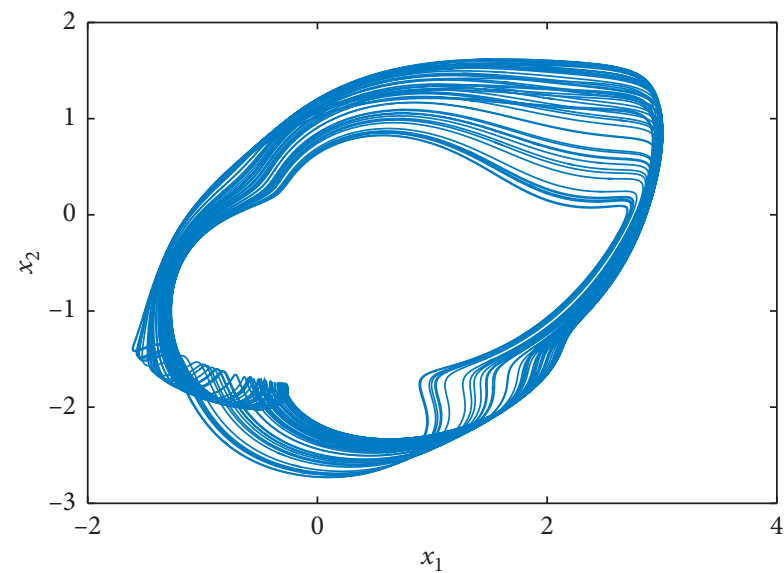

(a)

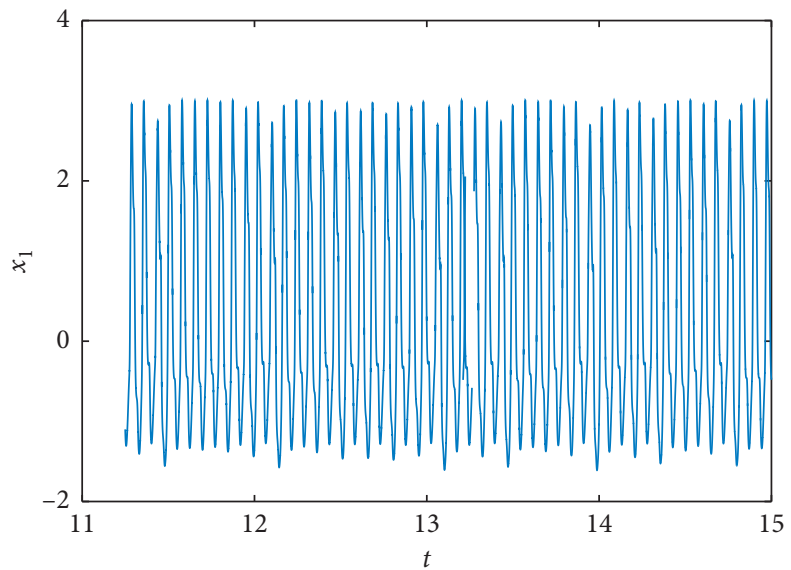

(b)

Figure 8: Phase portrait on plane $\left(x_{1}, x_{2}\right)$ and the waveforms on the plane $\left(t, x_{1}\right)$ of the first-order mode of the system with quasiperiodic motion when $P_{1}=52$. 


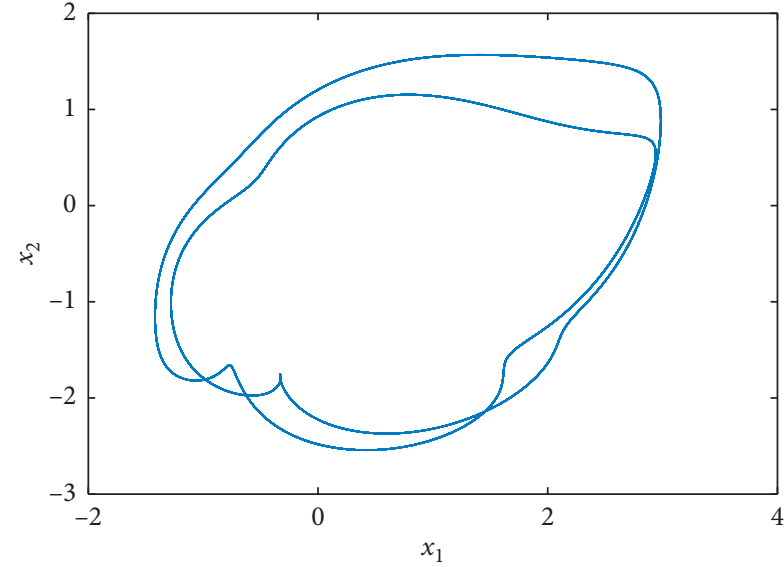

(a)

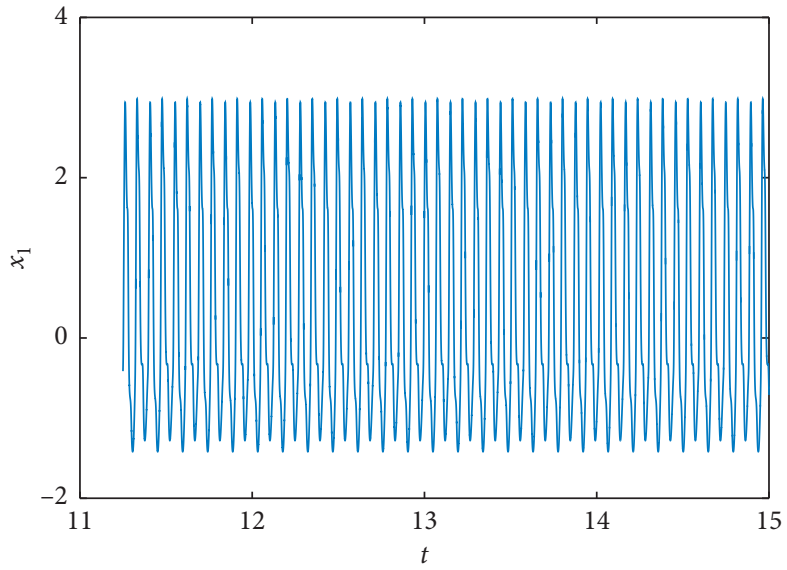

(b)

FIGURE 9: Phase portrait on plane $\left(x_{1}, x_{2}\right)$ and the waveforms on the plane $\left(t, x_{1}\right)$ of the first-order mode of the system with periodic-2 motion when $P_{1}=57$.

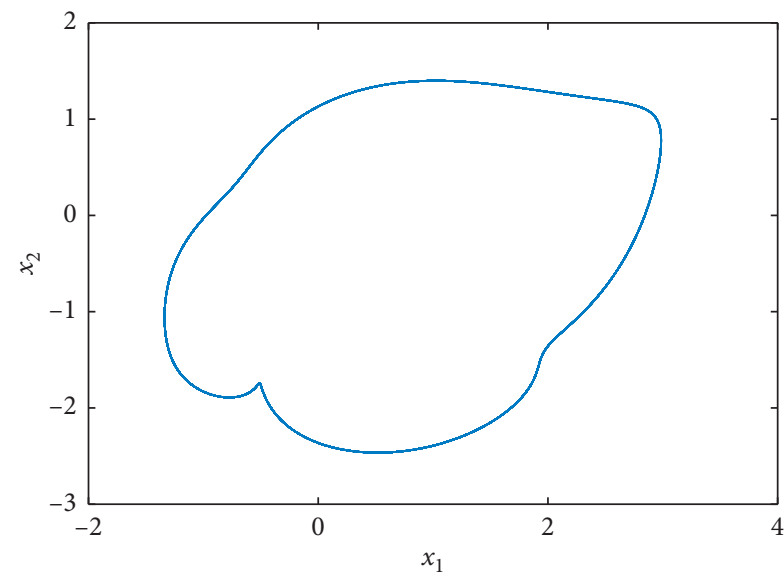

(a)

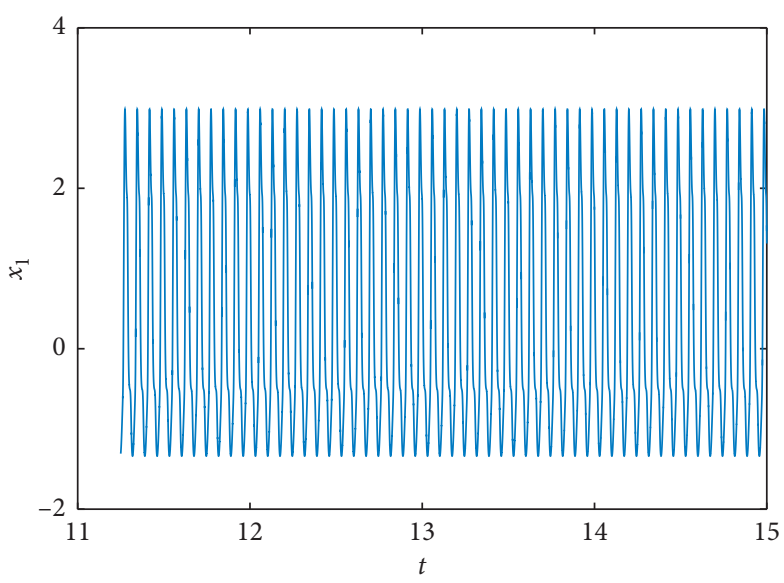

(b)

Figure 10: Phase portrait on plane $\left(x_{1}, x_{2}\right)$ and the waveforms on the plane $\left(t, x_{1}\right)$ of the first-order mode of the system with periodic-1 motion when $P_{1}=63$.

Similarly, the Runge-Kutta algorithm is utilized for the numerical simulation of the average equations (28) of the second-order response, and the initial value is selected as $x_{30}=1.4, x_{40}=1.75$. By choosing the excitation amplitude as the control parameter, the global bifurcation diagram of the second mode is obtained in Figure 11.

Figure 11 is the global bifurcation diagram of the second mode of the subharmonic vibration of rectangular laminated plates. It can be seen from the figure that as the amplitude of the external excitation increases, the vibration response of the second mode also presents different vibration forms. When the amplitude of external excitation is small, the vibration response of the system is chaotic. With the increase in the amplitude of external excitation, the system changes from chaotic motion to period-3 motion and then from period-3 motion to chaotic motion again. Figures 12-14 show the waveform and phase diagrams of the second-order modes of the subharmonic vibration of laminated plates with different excitation amplitudes. 


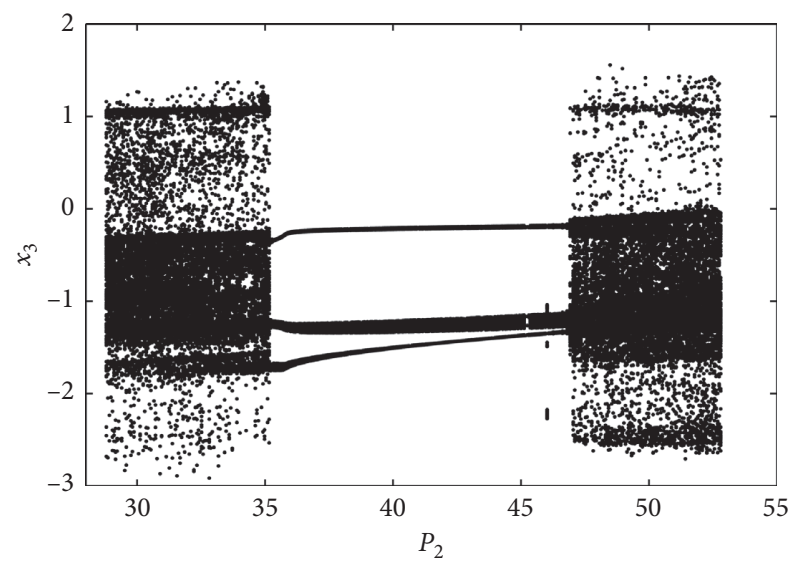

FIgURE 11: Bifurcation diagram for the second-order mode via external excitation.

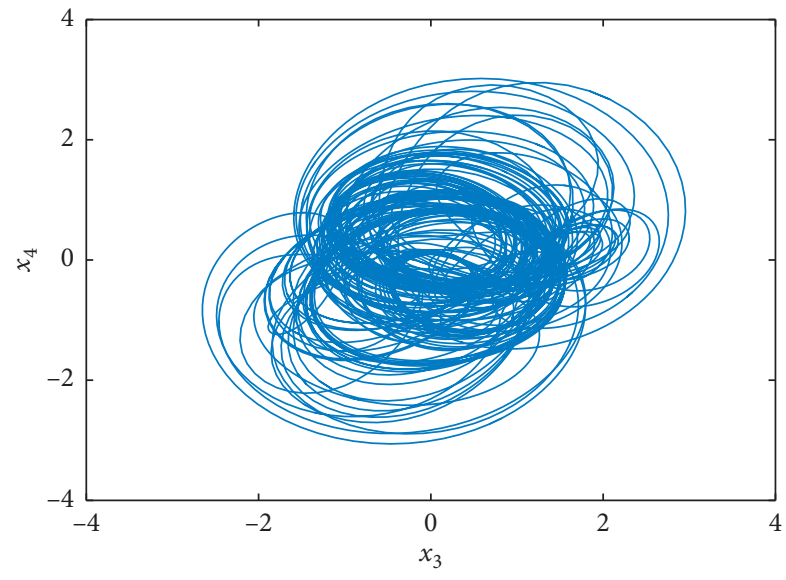

(a)

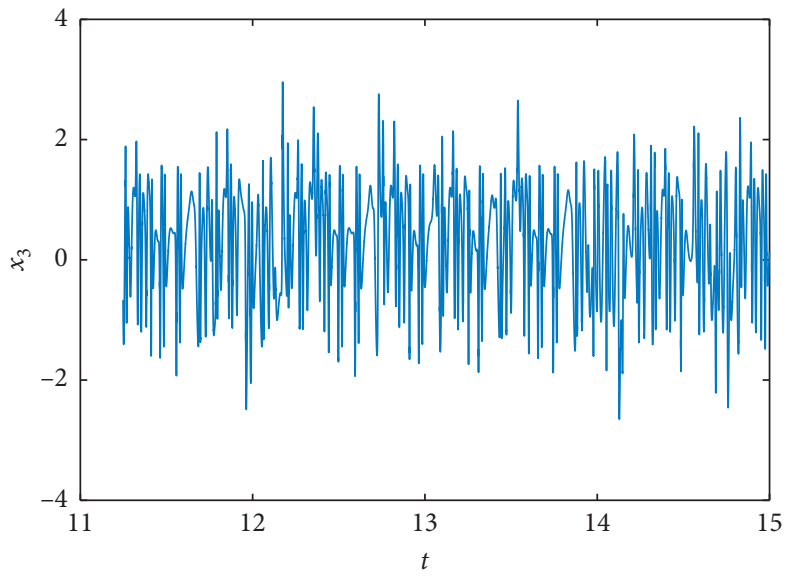

(b)

FIGURE 12: Phase portrait on plane $\left(x_{3}, x_{4}\right)$ and the waveforms on the plane $\left(t, x_{3}\right)$ of the second-order mode of the system with chaotic motion when $P_{1}=32$.

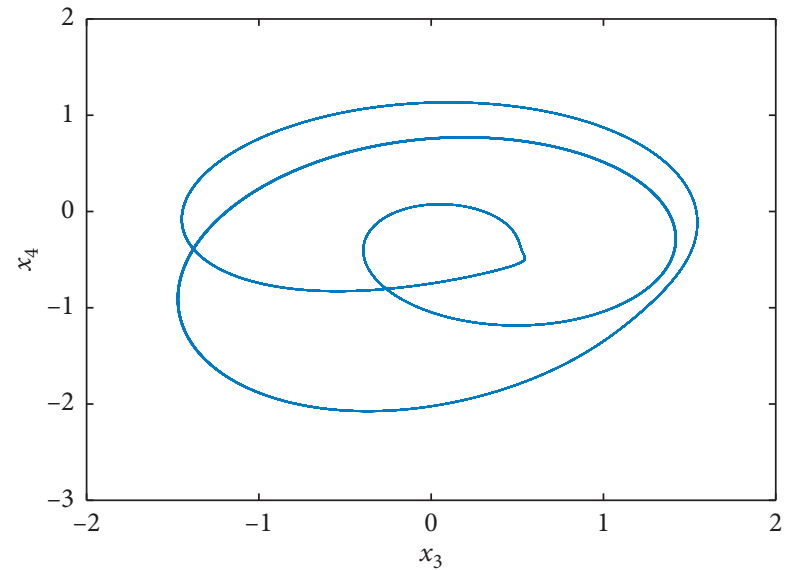

(a)

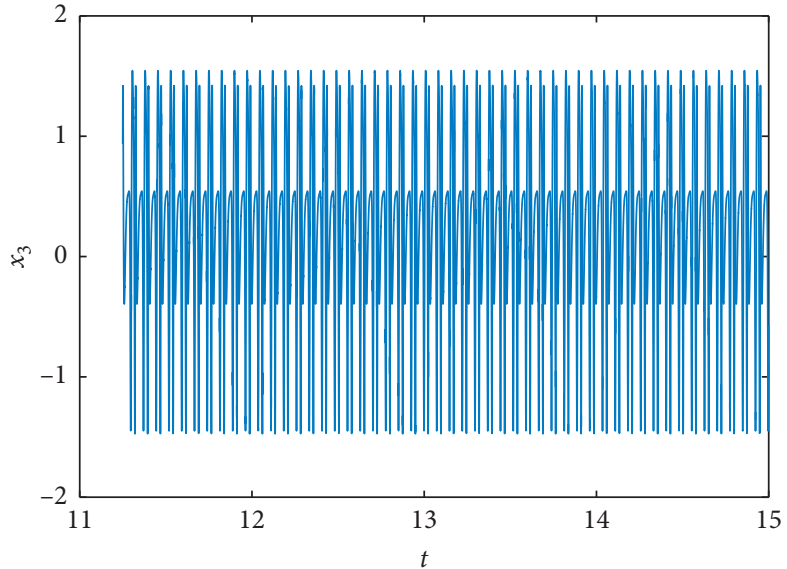

(b)

Figure 13: Phase portrait on plane $\left(x_{3}, x_{4}\right)$ and the waveforms on the plane $\left(t, x_{3}\right)$ of the second-order mode of the system with periodic- 3 motion when $P_{1}=42$. 


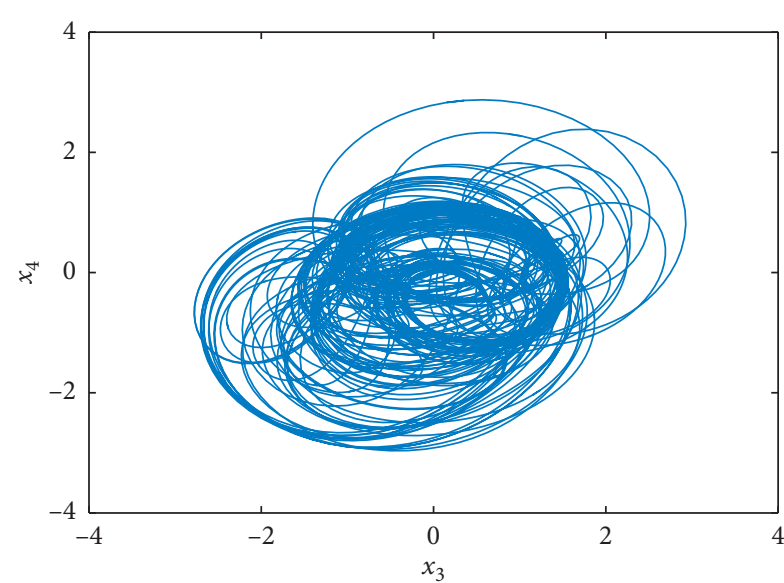

(a)

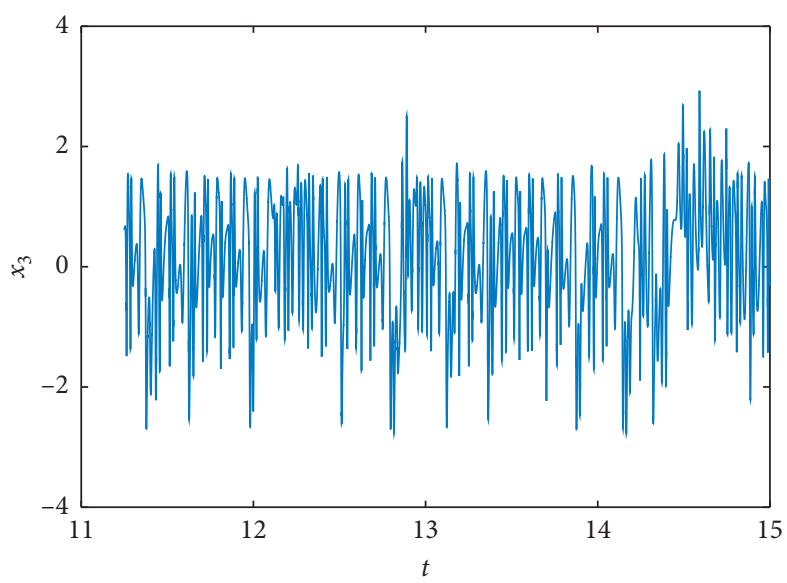

(b)

Figure 14: Phase portrait on plane $\left(x_{3}, x_{4}\right)$ and the waveforms on the plane $\left(t, x_{3}\right)$ of the second-order mode of the system with chaotic motion when $P_{1}=52$.

\section{Conclusions}

In this work, the subharmonic resonance of a rectangular laminated plate under harmonic excitation is studied. The vibration equations of the system are established using von Karman's nonlinear geometric relation and Hamilton's principle. The amplitude-frequency equations and the average equations in rectangular coordinates are obtained by using the multiscale method. The amplitude-frequency equations and average equations are numerically simulated in order to obtain the influence of system parameters on the nonlinear characteristics of vibration. The following conclusions can be obtained:

(1) The results show that there are many similar characteristics in the nonlinear response of the uncoupled two-order modes, which are excited separately when subharmonic resonance occurs. Under the same amplitude of external excitation, the amplitudes of the two-order modes increase as the excitation frequency increases along with both the subharmonic domains. For the same excitation frequency, the steady-state solutions corresponding to the large amplitudes are almost independent of the external excitation. The difference is that the steady-state solution with smaller amplitude of the first-order mode increases with the increase in the excitation amplitude, while that of the second-order mode decreases with the increase in the excitation amplitude.

(2) From the bifurcation diagram, it can be concluded that the system may experience many kinds of vibration states with the change in the amplitude of external excitation, such as quasiperiodic, periodic, and chaotic motion. The vibration of laminated plates can be controlled by adjusting the amplitude of the external excitation.

\section{Data Availability}

The data used to support the findings of this study are included within the article.

\section{Conflicts of Interest}

The authors declare that there are no conflicts of interest.

\section{Acknowledgments}

The authors sincerely acknowledge the financial support of the National Natural Science Foundation of China (Grants nos. 11862020, 11962020, and 11402126) and the Inner Mongolia Natural Science Foundation (Grants nos. 2018LH01014 and 2019MS05065).

\section{References}

[1] M. Wang, Z.-M. Li, and P. Qiao, "Vibration analysis of sandwich plates with carbon nanotube-reinforced composite face-sheets," Composite Structures, vol. 200, pp. 799-809, 2018.

[2] S. Razavi and A. Shooshtari, "Nonlinear free vibration of magneto-electro-elastic rectangular plates," Composite Structures, vol. 119, pp. 377-384, 2015.

[3] M. Sadri and D. Younesian, "Nonlinear harmonic vibration analysis of a plate-cavity system," Nonlinear Dynamics, vol. 74, no. 4, pp. 1267-1279, 2013.

[4] D. Aranda-Iiglesias, J. A. Rodríguez-Martínez, and M. B. Rubin, "Nonlinear axisymmetric vibrations of a hyperelastic orthotropic cylinder," International Journal of Non Linear Mechanics, vol. 99, pp. 131-143, 2018.

[5] J. Torabi and R. Ansari, "Nonlinear free vibration analysis of thermally induced FG-CNTRC annular plates: asymmetric versus axisymmetric study," Computer Methods in Applied Mechanics and Engineering, vol. 324, pp. 327-347, 2017.

[6] P. Ribeiro and M. Petyt, "Non-linear free vibration of isotropic plates with internal resonance," International Journal of Non-linear Mechanics, vol. 35, no. 2, pp. 263-278, 2000.

[7] H. Asadi, M. Bodaghi, M. Shakeri, and M. M. Aghdam, "Nonlinear dynamics of SMA-fiber-reinforced composite beams subjected to a primary/secondary-resonance excitation," Acta Mechanica, vol. 226, no. 2, pp. 437-455, 2015.

[8] Y. Zhang and Y. Li, "Nonlinear dynamic analysis of a double curvature honeycomb sandwich shell with simply supported 
boundaries by the homotopy analysis method," Composite Structures, vol. 221, p. 110884, 2019.

[9] J. N. Reddy, Mechanics of Laminated Composite Plates Theory and Analysis, CRC Press, Boca Raton, FL, USA, 2004.

[10] Y.-C. Lin and C.-C. Ma, "Resonant vibration of piezoceramic plates in fluid," Interaction and Multiscale Mechanics, vol. 1, no. 2, pp. 177-190, 2008.

[11] I. D. Breslavsky, M. Amabili, and M. Legrand, "Nonlinear vibrations of thin hyperelastic plates," Journal of Sound and Vibration, vol. 333, no. 19, pp. 4668-4681, 2014.

[12] A. A. Khdeir and J. N. Reddy, "On the forced motions of antisymmetric cross-ply laminated plates," International Journal of Mechanical Sciences, vol. 31, no. 7, pp. 499-510, 1989.

[13] P. Litewka and R. Lewandowski, "Nonlinear harmonically excited vibrations of plates with zener material," Nonlinear Dynamics, vol. 89, pp. 1-22, 2017.

[14] H. Eslami and O. A. Kandil, "Two-mode nonlinear vibration of orthotropic plates using method of multiple scales," Aiaa Journal, vol. 27, pp. 961-967, 2012.

[15] M. Amabili, "Nonlinear damping in nonlinear vibrations of rectangular plates: derivation from viscoelasticity and experimental validation," Journal of the Mechanics and Physics of Solids, vol. 118, pp. 275-292, 2018.

[16] M. Delapierre, S. H. Lohaus, and S. Pellegrino, "Nonlinear vibration of transversely-loaded spinning membranes," Journal of Sound and Vibration, vol. 427, pp. 41-62, 2018.

[17] S. Kumar, A. Mitra, and H. Roy, "Forced vibration response of axially functionally graded non-uniform plates considering geometric nonlinearity," International Journal of Mechanical Sciences, vol. 128-129, pp. 194-205, 2017.

[18] C.-S. Chen, C.-P. Fung, and R.-D. Chien, "A further study on nonlinear vibration of initially stressed plates," Applied Mathematics and Computation, vol. 172, no. 1, pp. 349-367, 2006.

[19] P. Balasubramanian, G. Ferrari, M. Amabili, and Z. J. G. N. del Prado, "Experimental and theoretical study on large amplitude vibrations of clamped rubber plates," International Journal of Non-linear Mechanics, vol. 94, pp. 36-45, 2017.

[20] W. Zhang, Y. X. Hao, and J. Yang, "Nonlinear dynamics of FGM circular cylindrical shell with clamped-clamped edges," Composite Structures, vol. 94, no. 3, pp. 1075-1086, 2012.

[21] J. A. Bennett, "Nonlinear vibration of simply supported angle ply laminated plates," AIAA Journal, vol. 9, no. 10, pp. 1997-2003, 1971.

[22] M. Rafiee, M. Mohammadi, B. Sobhani Aragh, and H. Yaghoobi, "Nonlinear free and forced thermo-electroaero-elastic vibration and dynamic response of piezoelectric functionally graded laminated composite shells," Composite Structures, vol. 103, pp. 188-196, 2013.

[23] S. C. Kattimani, "Geometrically nonlinear vibration analysis of multiferroic composite plates and shells," Composite Structures, vol. 163, pp. 185-194, 2017.

[24] N. D. Duc, P. H. Cong, and V. D. Quang, "Nonlinear dynamic and vibration analysis of piezoelectric eccentrically stiffened FGM plates in thermal environment," International Journal of Mechanical Sciences, vol. 115-116, pp. 711-722, 2016.

[25] N. Mohamed, M. A. Eltaher, S. A. Mohamed, and L. F. Seddek, "Numerical analysis of nonlinear free and forced vibrations of buckled curved beams resting on nonlinear elastic foundations," International Journal of Non-linear Mechanics, vol. 101, pp. 157-173, 2018.

[26] D. S. Cho, J.-H. Kim, T. M. Choi, B. H. Kim, and N. Vladimir, "Free and forced vibration analysis of arbitrarily supported rectangular plate systems with attachments and openings," Engineering Structures, vol. 171, pp. 1036-1046, 2018.

[27] A. H. Nayfeh and D. T. Mook, Nonlinear Oscillations, Wiley, Hoboken, NJ, USA, 1979.

[28] W. Zhang and M. H. Zhao, "Nonlinear vibrations of a composite laminated cantilever rectangular plate with one-toone internal resonance," Nonlinear Dynamics, vol. 70, no. 1, pp. 295-313, 2012.

[29] Y. F. Zhang, W. Zhang, and Z. G. Yao, "Analysis on nonlinear vibrations near internal resonances of a composite laminated piezoelectric rectangular plate," Engineering Structures, vol. 173, pp. 89-106, 2018.

[30] X. Y. Guo and W. Zhang, "Nonlinear vibrations of a reinforced composite plate with carbon nanotubes," Composite Structures, vol. 135, pp. 96-108, 2016.

[31] S. I. Chang, J. M. Lee, A. K. Bajaj, and C. M. Krousgrill, "Subharmonic responses in harmonically excited rectangular plates with one-to-one internal resonance," Chaos, Solitons \& Fractals, vol. 8, no. 4, pp. 479-498, 1997.

[32] D.-B. Zhang, Y.-Q. Tang, H. Ding, and L.-Q. Chen, "Parametric and internal resonance of a transporting plate with a varying tension," Nonlinear Dynamics, vol. 98, no. 4, pp. 2491-2508, 2019.

[33] S. W. Yang, W. Zhang, Y. X. Hao, and Y. Niu, "Nonlinear vibrations of FGM truncated conical shell under aerodynamics and in-plane force along meridian near internal resonances," Thin-walled Structures, vol. 142, pp. 369-391, 2019.

[34] Y. D. Hu and J. Li, "The magneto-elastic subharmonic resonance of current-conducting thin plate in magnetic field," Journal of Sound \& Vibration, vol. 319, pp. 1107-1120, 2009.

[35] F.-M. Li and G. Yao, "1/3 Subharmonic resonance of a nonlinear composite laminated cylindrical shell in subsonic air flow," Composite Structures, vol. 100, pp. 249-256, 2013.

[36] E. Jomehzadeh, A. R. Saidi, Z. Jomehzadeh et al., "Nonlinear subharmonic oscillation of orthotropic graphene-matrix composite," Computational Materials Science, vol. 99, pp. 164-172, 2015.

[37] M. R. Permoon, H. Haddadpour, and M. Javadi, "Nonlinear vibration of fractional viscoelastic plate: primary, subharmonic, and superharmonic response," International Journal of Non-linear Mechanics, vol. 99, pp. 154-164, 2018.

[38] H. Ahmadi and K. Foroutan, "Nonlinear vibration of stiffened multilayer FG cylindrical shells with spiral stiffeners rested on damping and elastic foundation in thermal environment," Thin-Walled Structures, vol. 145, pp. 106-116, 2019.

[39] S. M. Hosseini, A. Shooshtari, H. Kalhori, and S. N. Mahmoodi, "Nonlinear-forced vibrations of piezoelectrically actuated viscoelastic cantilevers," Nonlinear Dynamics, vol. 78, no. 1, pp. 571-583, 2014.

[40] J. Náprstek and C. Fischer, "Super and sub-harmonic synchronization in generalized van der Pol oscillator," Computers \& Structures, vol. 224, Article ID 106103, 2019. 